\title{
CENTRAL EXTENSIONS AND STOCHASTIC PROCESSES ASSOCIATED WITH THE LIE ALGEBRA OF THE RENORMALIZED HIGHER POWERS OF WHITE NOISE
}

\author{
LUIGI ACCARDI \\ Centro Vito Volterra, Università di Roma Tor Vergata \\ via Columbia 2, 00133 Roma, Italy \\ E-mail:volterra@volterra.mat.uniroma2.it \\ http://volterra.mat.uniroma2.it \\ ANDREAS BOUKAS \\ Department of Mathematics and Natural Sciences, American College of Greece \\ Aghia Paraskevi 15342, Athens, Greece \\ E-mail: andreasboukas@acgmail.gr
}

\begin{abstract}
In the first part of the paper we discuss possible definitions of Fock representation of the $*$-Lie algebra of the Renormalized Higher Powers of White Noise $(R H P W N)$. We propose one definition that avoids the no-go theorems and we show that the vacuum distribution of the analogue of the field operator for the $n$-th renormalized power of WN defines a continuous binomial process. In the second part of the paper we present without proof our recent results on the central extensions of $R H P W N$, its subalgebras and the $w_{\infty}$ Lie algebra of conformal field theory. In the third part of the paper we describe our results on the non-trivial central extensions of the Heisenberg algebra. This is a 4-dimensional Lie algebra, hence belonging to a list which is well known and has been studied by several research groups. However the canonical nature of this algebra, i.e. the fact that it is the unique (up to a complex scaling) non-trivial central extension of the Heisenberg algebra, seems to be new. We also find the possible vacuum distributions corresponding to a family of injective $*$-homomorphisms of different non-trivial central extensions of the Heisenberg algebra into the Schrödinger algebra.
\end{abstract}

2000 Mathematics Subject Classification: 60H40, 81S05, 81T30, 81T40.

Key words and phrases: renormalized powers of white noise, second quantization, Heisenberg algebra, $w_{\infty}$-algebra, Virasoro algebra, Zamolodchikov algebra, Fock space, moment systems, continuous binomial distribution, central extension, group law.

The paper is in final form and no version of it will be published elsewhere. 
1. The $R H P W N$ and Virasoro-Zamolodchikov- $w_{\infty} *$-Lie algebras. Let $a_{t}$ and $a_{s}^{\dagger}$ be the standard boson white noise functionals with commutator

$$
\left[a_{t}, a_{s}^{\dagger}\right]=\delta(t-s) \cdot 1
$$

where $\delta$ is the Dirac delta function. As shown in [1] and [2, using the renormalization

$$
\delta^{l}(t-s)=\delta(s) \delta(t-s), \quad l=2,3, \ldots
$$

for the higher powers of the Dirac delta function and choosing test functions $f: \mathbb{R} \rightarrow \mathbb{C}$ that vanish at zero, the symbols

$$
B_{k}^{n}(f)=\int_{\mathbb{R}} f(s) a_{s}^{\dagger^{n}} a_{s}^{k} d s, \quad n, k \in\{0,1,2, \ldots\}
$$

with involution

$$
\left(B_{k}^{n}(f)\right)^{*}=B_{n}^{k}(\bar{f})
$$

and

$$
B_{0}^{0}(f)=\int_{\mathbb{R}} f(s) d s
$$

satisfy the Renormalized Higher Powers of White Noise $(R H P W N)$ commutation relations

$$
\left[B_{k}^{n}(g), B_{K}^{N}(f)\right]_{R H P W N}=(k N-K n) B_{k+K-1}^{n+N-1}(g f)
$$

where for $n<0$ and/or $k<0$ we define $B_{k}^{n}(f)=0$. Moreover, for $n, N \geq 2$ and $k, K \in \mathbb{Z}$ the white noise operators

$$
\hat{B}_{k}^{n}(f)=\int_{\mathbb{R}} f(t) e^{\frac{k}{2}\left(a_{t}-a_{t}^{\dagger}\right)}\left(\frac{a_{t}+a_{t}^{\dagger}}{2}\right)^{n-1} e^{\frac{k}{2}\left(a_{t}-a_{t}^{\dagger}\right)} d t
$$

satisfy the commutation relations

$$
\left[\hat{B}_{k}^{n}(g), \hat{B}_{K}^{N}(f)\right]_{w_{\infty}}=((N-1) k-(n-1) K) \hat{B}_{k+K}^{n+N-2}(g f)
$$

of the Virasoro-Zamolodchikov- $w_{\infty}$ Lie algebra of conformal field theory with involution

$$
\left(\hat{B}_{k}^{n}(f)\right)^{*}=\hat{B}_{-k}^{n}(\bar{f})
$$

In particular, for $n=N=2$ we obtain

$$
\left[\hat{B}_{k}^{2}(g), \hat{B}_{K}^{2}(f)\right]_{w_{\infty}}=(k-K) \hat{B}_{k+K}^{2}(g f)
$$

which are the commutation relations of the Virasoro algebra. The analytic continuation $\left\{\hat{B}_{z}^{n}(f) ; n \geq 1, z \in \mathbb{C}\right\}$ of the Virasoro-Zamolodchikov- $w_{\infty}$ Lie algebra and the $R H P W N$ Lie algebra with commutator $[\cdot, \cdot]_{R H P W N}$ have recently been identified (cf. [3]) providing a connection between quantum probability and conformal field theory and high-energy physics.

The first five sections of this paper contain the full proofs of the results outlined in [5] with the remaining sections focusing on our results on central extensions. 
For a detailed exposition of the motivations behind the theory of the renormalized higher powers of white noise, as well as its historical development, we refer to [8].

Notation 1. In what follows, for all integers $n, k$ we will use the notation

$$
B_{k}^{n}=B_{k}^{n}\left(\chi_{I}\right)
$$

where $I$ is some fixed subset of $\mathbb{R}$ of finite Lebesgue measure $\mu=\mu(I)>0$.

2. The action of the $R H P W N$ operators on the Fock vacuum vector $\Phi$. Recall that our strategy to construct a Fock representation for the $R H P W N$ *-Lie-algebra is to define an action of its elements on a single vector (the vacuum) and then prove that this action uniquely determines a Hilbert space structure. From this point of view, Lemma 1 and Proposition 1 constitute a heuristic motivation for the action that we begin to investigate starting from Proposition 2.

2.1. Definition of the $R H P W N$ action on the Fock vacuum vector $\Phi$. To formulate a reasonable definition of the action of the $R H P W N$ operators on $\Phi$ we go to the level of white noise. As it will turn out, $\Phi$ must be an eigenvector of the number operators $B_{n}^{n}(f)$. The following is a continuous, non-renormalized version of a known formula in the finite dimensional case. We use it as motivation for the identification proved in Proposition 1 .

Lemma 1. For all $t \geq s \geq 0$ and $n \in\{0,1,2, \ldots\}$

$$
\left(a_{t}^{\dagger}\right)^{n}\left(a_{s}\right)^{n}=\sum_{k=0}^{n} s_{n, k}\left(a_{t}^{\dagger} a_{s}\right)^{k} \delta^{n-k}(t-s)
$$

where $s_{n, k}$ are the Stirling numbers of the first kind with $s_{0,0}=1$ and $s_{0, k}=s_{n, 0}=0$ for all $n, k \geq 1$.

Idea of the proof. It is well known (see [4] for a proof) that if $\left[b, b^{\dagger}\right]=1$ then

$$
\left(b^{\dagger}\right)^{k}(b)^{k}=\sum_{m=0}^{k} s_{k, m}\left(b^{\dagger} b\right)^{m}
$$

If $\left[b, b^{\dagger}\right]=1$ we can represent $a_{t}^{\dagger}, a_{t}$ using the formal expressions

$$
\delta(t-s)^{1 / 2} b^{\dagger}=a_{t}^{\dagger}, \text { and } \delta(t-s)^{1 / 2} b=a_{s}
$$

Then the result follows by substituting 2.3 into 2.2 . The same result follows by induction using 1.1.

The following proposition shows that, if we combine the renormalization 1.2 with the identity (2.1) then we obtain a new expression for the renormalized powers $B_{k}^{n}(f)$.

Proposition 1. For all integers $n \geq k \geq 0$ and for all test functions $f$

$$
B_{k}^{n}(f)=\int_{\mathbb{R}} f(t)\left(a_{t}^{\dagger}\right)^{n-k}\left(a_{t}^{\dagger} a_{t}\right)^{k} d t
$$

Proof. For $n \geq k$ we can write

$$
\left(a_{t}^{\dagger}\right)^{n}\left(a_{s}\right)^{k}=\left(a_{t}^{\dagger}\right)^{n-k}\left(a_{t}^{\dagger}\right)^{k}\left(a_{s}\right)^{k}
$$


Multiplying both sides by $f(t) \delta(t-s)$ and then taking $\int_{\mathbb{R}} \int_{\mathbb{R}} \ldots d s d t$ of both sides of the resulting equation we obtain

$$
\int_{\mathbb{R}} \int_{\mathbb{R}} f(t)\left(a_{t}^{\dagger}\right)^{n}\left(a_{s}\right)^{k} \delta(t-s) d s d t=\int_{\mathbb{R}} \int_{\mathbb{R}} f(t)\left(a_{t}^{\dagger}\right)^{n-k}\left(a_{t}^{\dagger}\right)^{k}\left(a_{s}\right)^{k} \delta(t-s) d s d t
$$

which, after applying (1.3) to its left and Lemma 1 to its right hand side, yields

$$
\begin{aligned}
B_{k}^{n}(f) & =\sum_{m=0}^{k} s_{k, m} \int_{\mathbb{R}} \int_{\mathbb{R}} f(t)\left(a_{t}^{\dagger}\right)^{n-k}\left(a_{t}^{\dagger} a_{s}\right)^{m} \delta^{k-m+1}(t-s) d s d t \\
& =s_{k, k} \int_{\mathbb{R}} \int_{\mathbb{R}} f(t)\left(a_{t}^{\dagger}\right)^{n-k}\left(a_{t}^{\dagger} a_{s}\right)^{k} \delta(t-s) d s d t \\
& +\sum_{m=0}^{k-1} s_{k, m} \int_{\mathbb{R}} \int_{\mathbb{R}} f(t)\left(a_{t}^{\dagger}\right)^{n-k}\left(a_{t}^{\dagger} a_{s}\right)^{m} \delta(s) \delta(t-s) d s d t \\
& =s_{k, k} \int_{\mathbb{R}} f(t)\left(a_{t}^{\dagger}\right)^{n-k}\left(a_{t}^{\dagger} a_{t}\right)^{k} d t+0 \\
& =\int_{\mathbb{R}} f(t)\left(a_{t}^{\dagger}\right)^{n-k}\left(a_{t}^{\dagger} a_{t}\right)^{k} d t
\end{aligned}
$$

where we have used the renormalization rule $[1.2), f(0)=0$, and $s_{k, k}=1$.

Proposition 2. Suppose that there exist a Hilbert space $H$, a representation of the RHPWN *-Lie-algebra on $H$ (omitted for simplicity from the notations) and a vector $\Phi \in H$ such that for all $n, k \in\{0,1,2, \ldots\}$ and test functions $f$,

$$
B_{k}^{n}(f) \Phi= \begin{cases}0 & \text { if } n<k \text { or } n \cdot k<0, \\ B_{0}^{n-k}\left(f \sigma_{k}\right) \Phi & \text { if } n>k \geq 0, \\ \int_{\mathbb{R}} f(t) \rho_{k}(t) d t \Phi & \text { if } n=k,\end{cases}
$$

where $\sigma_{k}$ and $\rho_{k}$ are complex valued functions. Then there exist a number $\sigma_{1} \in \mathbb{C}$ such that, for all $n \in\{0,1,2, \ldots\}$ :

$$
\begin{gathered}
\sigma_{n}=\sigma_{1}^{n}, \\
\rho_{n}=\frac{\sigma_{1}^{n}}{n+1} .
\end{gathered}
$$

Proof. By 2.5 and (1.3) for $k=0$, and by (1.5) for $n=k=0$ it follows that $\sigma_{0}=\rho_{0}=1$. For $n \geq 1$ we have

$$
\begin{aligned}
\left\langle B_{0}^{n}(f) \Phi, B_{1}^{n+1}(g) \Phi\right\rangle & =\left\langle B_{0}^{n}(f) \Phi, B_{0}^{n}\left(g \sigma_{1}\right) \Phi\right\rangle \\
& =\left\langle\Phi, B_{n}^{0}(\bar{f}) B_{0}^{n}\left(g \sigma_{1}\right) \Phi\right\rangle \\
& =\left\langle\Phi,\left(B_{0}^{n}\left(g \sigma_{1}\right) B_{n}^{0}(\bar{f})+\left[B_{n}^{0}(\bar{f}), B_{0}^{n}\left(g \sigma_{1}\right)\right]\right) \Phi\right\rangle \\
& \left.=\left\langle\Phi,\left(0+n^{2} B_{n-1}^{n-1}\left(\bar{f} g \sigma_{1}\right)\right]\right) \Phi\right\rangle \\
& =n^{2} \int_{\mathbb{R}} \rho_{n-1}(t) \sigma_{1}(t) \bar{f}(t) g(t) d t
\end{aligned}
$$

and also 


$$
\begin{aligned}
\left\langle B_{0}^{n}(f) \Phi, B_{1}^{n+1}(g) \Phi\right\rangle & =\left\langle\Phi, B_{n}^{0}(\bar{f}) B_{1}^{n+1}(g) \Phi\right\rangle \\
& =\left\langle\Phi,\left(B_{1}^{n+1}(g) B_{n}^{0}(\bar{f})+\left[B_{n}^{0}(\bar{f}), B_{1}^{n+1}(g)\right]\right) \Phi\right\rangle \\
& \left.=\left\langle\Phi,\left(0+n(n+1) B_{n}^{n}(\bar{f} g)\right]\right) \Phi\right\rangle \\
& =n(n+1) \int_{\mathbb{R}} \rho_{n}(t) \bar{f}(t) g(t) d t,
\end{aligned}
$$

i.e., for all test functions $h$

$$
n^{2} \int_{\mathbb{R}} \rho_{n-1}(t) \sigma_{1}(t) h(t) d t=n(n+1) \int_{\mathbb{R}} \rho_{n}(t) h(t) d t
$$

which implies that

$$
\rho_{n}=\frac{n}{n+1} \sigma_{1} \rho_{n-1}=\ldots=\frac{\sigma_{1}^{n}}{n+1}
$$

thus proving (2.7). Similarly,

$$
\begin{aligned}
\int_{\mathbb{R}} \rho_{n}(t) f(t) g(t) d t & =\left\langle\Phi, B_{n}^{n}(f g) \Phi\right\rangle=\frac{1}{n+1}\left\langle\Phi,\left[B_{n}^{n-1}(f), B_{1}^{2}(g)\right] \Phi\right\rangle \\
& =\frac{1}{n+1}\left\langle\Phi,\left(B_{n}^{n-1}(f) B_{1}^{2}(g)-B_{1}^{2}(g) B_{n}^{n-1}(f)\right) \Phi\right\rangle \\
& =\frac{1}{n+1}\left\langle\Phi, B_{n}^{n-1}(f) B_{1}^{2}(g) \Phi\right\rangle=\frac{1}{n+1}\left\langle B_{n-1}^{n}(\bar{f}) \Phi, B_{1}^{2}(g) \Phi\right\rangle \\
& =\frac{1}{n+1}\left\langle B_{0}^{1}\left(\sigma_{n-1} \bar{f}\right) \Phi, B_{0}^{1}\left(\sigma_{1} g\right) \Phi\right\rangle=\frac{1}{n+1}\left\langle\Phi, B_{1}^{0}\left(\bar{\sigma}_{n-1} f\right) B_{0}^{1}\left(\sigma_{1} g\right) \Phi\right\rangle \\
& =\frac{1}{n+1}\left\langle\Phi,\left[B_{1}^{0}\left(\bar{\sigma}_{n-1} f\right) B_{0}^{1}\left(\sigma_{1} g\right)\right] \Phi\right\rangle=\frac{1}{n+1}\left\langle\Phi, B_{0}^{0}\left(\bar{\sigma}_{n-1} f \sigma_{1} g\right) \Phi\right\rangle \\
& =\frac{1}{n+1} \int_{\mathbb{R}} \bar{\sigma}_{n-1}(t) \sigma_{1}(t) f(t) g(t) d t .
\end{aligned}
$$

Thus, for all test functions $h$

$$
\int_{\mathbb{R}} \rho_{n}(t) h(t) d t=\frac{1}{n+1} \int_{\mathbb{R}} \bar{\sigma}_{n-1}(t) \sigma_{1}(t) h(t) d t
$$

therefore

$$
(n+1) \rho_{n}=\bar{\sigma}_{n-1} \sigma_{1}
$$

which combined with 2.7 implies $\bar{\sigma}_{n-1}=\sigma_{1}^{n-1}$, which in turn implies that the $\sigma_{n}$ 's are real and yields 2.6 .

We want to keep the interpretation of $a_{t}^{\dagger}, a_{t}$ and $a_{t}^{\dagger} a_{t}$ as creation, annihilation and number densities respectively even after their renormalization. From this point of view, because of the identity (2.4), the first condition in definition 2.5 of the action of $B_{k}^{n}$ on $\Phi$, is quite natural. The second condition is equivalent to assuming that $\Phi$ is an eigenvector of the number density with eigenvalue $\sigma_{k}(t)$ and the third condition allows for a different action of the number density when it appears as a pure number density or when it multiplies a non zero power of the creation density. In the following we take the function $\sigma_{1}$ (and thus by 2.6 all the $\sigma_{n}$ 's ) appearing in Proposition 2 to be identically equal to 1 and we arrive to the following definition of the action of the $R H P W N$ operators on $\Phi$. 
Definition 1. For $n, k \in \mathbb{Z}$ and test functions $f$

$$
B_{k}^{n}(f) \Phi= \begin{cases}0 & \text { if } n<k \text { or } n \cdot k<0, \\ B_{0}^{n-k}(f) \Phi & \text { if } n>k \geq 0, \\ \frac{1}{n+1} \int_{\mathbb{R}} f(t) d t \Phi & \text { if } n=k .\end{cases}
$$

\subsection{The $n$-th order $R H P W N$ *-Lie algebras $\mathcal{L}_{n}$}

DEFINITION 2. In the notation (1.11):

(i) $\mathcal{L}_{1}$ is the $*$-Lie algebra generated by $B_{0}^{1}$ and $B_{1}^{0}$, i.e. the linear span of $\left\{B_{0}^{1}, B_{1}^{0}, B_{0}^{0}\right\}$.

(ii) $\mathcal{L}_{2}$ is the $*$-Lie algebra generated by $B_{0}^{2}$ and $B_{2}^{0}$, i.e. the linear span of $\left\{B_{0}^{2}, B_{2}^{0}, B_{1}^{1}\right\}$.

(iii) For $n \in\{3,4, \ldots\}, \mathcal{L}_{n}$ is the $*$-Lie algebra generated by $B_{0}^{n}$ and $B_{n}^{0}$ through repeated commutations and linear combinations. It consists of linear combinations of creation/annihilation operators of the form $B_{y}^{x}$ where $x-y=k n, k \in \mathbb{Z} \backslash\{0\}$, and of number operators $B_{x}^{x}$ with $x \geq n-1$.

REMARK. In particular, choosing $x=n, k=2, y=0$ in (iii) of Definition 2, one sees that, for each $n \geq 3, \mathcal{L}_{n}$ contains also $B_{0}^{2 n}$. As shown in the following section, this is the root of the no-go theorems.

2.3. The Fock representation no-go theorem. We will show that if the $R H P W N$ action on $\Phi$ is that of Definition 1 then the Fock representation no-go theorems of [9] and 2] can be extended to the $R H P W N *$-Lie algebras $\mathcal{L}_{n}$ where $n \geq 3$.

THEOREM 1. Let $n \geq 3$. If the action of the RHPWN operators on the vacuum vector $\Phi$ is given by Definition 1 then $\mathcal{L}_{n}$ does not admit a Fock representation whenever the test function space contains characteristic functions of Lebesgue measure strictly smaller than $n^{2}(n+1) / 2$.

Proof. From the remark after Definition 2 we know that, for each $n \geq 3, \mathcal{L}_{n}$ contains $B_{0}^{2 n}$. Therefore, in the assumptions of the theorem, one must have, $\forall a, b \in \mathbb{C}$ :

$$
0 \leq\left\|\left(a B_{0}^{2 n}+b\left(B_{0}^{n}\right)^{2}\right) \Phi\right\|=\left\langle\left(a B_{0}^{2 n}+b\left(B_{0}^{n}\right)^{2}\right) \Phi,\left(a B_{0}^{2 n}+b\left(B_{0}^{n}\right)^{2}\right) \Phi\right\rangle
$$

and, in the notation (1.11), the RHPWN operators are defined on the same interval $I$. Using the notation $\langle x\rangle=\langle\Phi, x \Phi\rangle$ this amounts to the positive semi-definiteness of the matrix

$$
A=\left[\begin{array}{cc}
\left\langle B_{2 n}^{0} B_{0}^{2 n}\right\rangle & \left\langle B_{2 n}^{0}\left(B_{0}^{n}\right)^{2}\right\rangle \\
\left\langle B_{2 n}^{0}\left(B_{0}^{n}\right)^{2}\right\rangle & \left\langle\left(B_{n}^{0}\right)^{2}\left(B_{0}^{n}\right)^{2}\right\rangle
\end{array}\right] .
$$

Using (1.8) and Definition 1 we find that

$$
\left\langle B_{2 n}^{0} B_{0}^{2 n}\right\rangle=4 n^{2}\left\langle B_{2 n-1}^{2 n-1}\right\rangle=4 n^{2} \frac{1}{2 n} \mu(I)=2 n \mu(I)
$$

and

$$
\begin{aligned}
\left\langle B_{2 n}^{0}\left(B_{0}^{n}\right)^{2}\right\rangle & =\left\langle B_{0}^{2 n} \Phi,\left(B_{0}^{n}\right)^{2} \Phi\right\rangle=\left\langle B_{n}^{0} B_{0}^{2 n} \Phi, B_{0}^{n} \Phi\right\rangle \\
& =2 n^{2}\left\langle B_{n-1}^{2 n-1} \Phi, B_{0}^{n} \Phi\right\rangle=2 n^{2}\left\langle B_{0}^{n} \Phi, B_{0}^{n} \Phi\right\rangle \\
& =2 n^{2}\left\langle B_{n}^{0} B_{0}^{n}\right\rangle=2 n^{2} n^{2}\left\langle B_{n-1}^{n-1}\right\rangle \\
& =2 n^{4} \frac{1}{n} \mu(I)=2 n^{3} \mu(I)
\end{aligned}
$$


and also

$$
\begin{aligned}
\left\langle\left(B_{n}^{0}\right)^{2}\left(B_{0}^{n}\right)^{2}\right\rangle & =\left\langle B_{0}^{n} \Phi, B_{n}^{0}\left(B_{0}^{n}\right)^{2} \Phi\right\rangle=\left\langle B_{0}^{n} \Phi,\left(B_{n}^{0} B_{0}^{n}\right) B_{0}^{n} \Phi\right\rangle \\
& =\left\langle B_{0}^{n} \Phi,\left(B_{0}^{n} B_{n}^{0}+n^{2} B_{n-1}^{n-1}\right) B_{0}^{n} \Phi\right\rangle \\
& =\left\langle B_{0}^{n} \Phi, B_{0}^{n} B_{n}^{0} B_{0}^{n} \Phi\right\rangle+n^{2}\left\langle B_{0}^{n} \Phi, B_{n-1}^{n-1} B_{0}^{n} \Phi\right\rangle \\
& =\left\langle B_{n}^{0} B_{0}^{n} \Phi, B_{n}^{0} B_{0}^{n} \Phi\right\rangle+n^{2}\left\langle B_{0}^{n} \Phi,\left(B_{0}^{n} B_{n-1}^{n-1}+n(n-1) B_{n-2}^{2 n-2}\right) \Phi\right\rangle \\
& =n^{4}\left\langle B_{n-1}^{n-1} \Phi, B_{n-1}^{n-1} \Phi\right\rangle+n \mu(I)\left\langle B_{0}^{n} \Phi, B_{0}^{n} \Phi\right\rangle+n^{3}(n-1)\left\langle B_{0}^{n} \Phi, B_{n-2}^{2 n-2} \Phi\right\rangle \\
& =n^{2} \mu(I)^{2}+n \mu(I)\left\langle B_{n}^{0} B_{0}^{n}\right\rangle+n^{3}(n-1)\left\langle B_{n}^{0} B_{n-2}^{2 n-2}\right\rangle \\
& =n^{2} \mu(I)^{2}+n^{3} \mu(I)\left\langle B_{n-1}^{n-1}\right\rangle+n^{4}(n-1)(2 n-2)\left\langle B_{2 n-3}^{2 n-3}\right\rangle \\
& =n^{2} \mu(I)^{2}+n^{2} \mu(I)^{2}+n^{4}(n-1) \mu(I) \\
& =2 n^{2} \mu(I)^{2}+n^{4}(n-1) \mu(I) .
\end{aligned}
$$

Thus

$$
A=\left[\begin{array}{cc}
2 n \mu(I) & 2 n^{3} \mu(I) \\
2 n^{3} \mu(I) & 2 n^{2} \mu(I)^{2}+n^{4}(n-1) \mu(I)
\end{array}\right]
$$

is a symmetric matrix so it is positive semi-definite if and only if its minors are nonnegative. The minor determinants of $A$ are

$$
d_{1}=2 n \mu(I)
$$

which is always nonnegative, and

$$
d_{2}=2 n^{3} \mu(I)^{2}\left(2 \mu(I)-n^{2}-n^{3}\right)
$$

which is nonnegative if and only if $\mu(I) \geq n^{2}(n+1) / 2$. This proves the statement.

\section{The $n$-th order truncated $R H P W N$ (or $T R H P W N$ ) Fock space $\mathcal{F}_{n}$}

3.1. Truncation of the $R H P W N$ Fock kernels. The generic element of the $*$-Lie algebras $\mathcal{L}_{n}$ of Definition 2 is $B_{0}^{n}$. All other elements of $\mathcal{L}_{n}$ are obtained by taking adjoints, commutators, and linear combinations. It thus makes sense to consider $\left(B_{0}^{n}(f)\right)^{k} \Phi$ as basis vectors for the $n$-th particle space of the Fock space $\mathcal{F}_{n}$ associated with $\mathcal{L}_{n}$. A calculation of the "Fock kernel" $\left\langle\left(B_{0}^{n}\right)^{k} \Phi,\left(B_{0}^{n}\right)^{m} \Phi\right\rangle$ reveals that it is the terms containing $B_{0}^{2 n} \Phi$ that prevent the kernel from being positive definite. Since $\mathcal{L}_{1}$ and $\mathcal{L}_{2}$ do not contain $B_{0}^{2}$ and $B_{0}^{4}$ respectively, that problem exists for $n \geq 3$ only and the Fock spaces $\mathcal{F}_{1}$ and $\mathcal{F}_{2}$ are actually not truncated. In what follows we will compute the Fock kernels by applying Definition 1 and by truncating "singular" terms of the form

$$
\left\langle\left(B_{0}^{n}\right)^{k} \Phi,\left(B_{0}^{n}\right)^{m} B_{y}^{x} \Phi\right\rangle
$$

where $n k=n m+x-y$ and $x-y=2 n$, i.e. $k-m=2$. This amounts to truncating the action of the principal $\mathcal{L}_{n}$ number operator $B_{n-1}^{n-1}$ on the "number vectors" $\left(B_{0}^{n}\right)^{k} \Phi$ which by commutation relations 11.6 and Definition 1 is of the form

$$
B_{n-1}^{n-1}\left(B_{0}^{n}\right)^{k} \Phi=\left(\frac{\mu}{n}+k n(n-1)\right)\left(B_{0}^{n}\right)^{k} \Phi+\sum_{i \geq 1} \prod_{j \geq 1} c_{i, j} B_{0}^{\lambda_{i, j} n} \Phi
$$


(where for each $i$ not all positive integers $\lambda_{i, j}$ are equal to 1 ) by omitting the $\sum_{i \geq 1} \prod_{j \geq 1} c_{i, j} B_{0}^{\lambda_{i, j} n} \Phi$ part. We thus arrive at the following:

Definition 3. For integers $n \geq 1$ and $k \geq 0$,

$$
B_{n-1}^{n-1}\left(B_{0}^{n}\right)^{k} \Phi:=\left(\frac{\mu}{n}+k n(n-1)\right)\left(B_{0}^{n}\right)^{k} \Phi,
$$

i.e., the number vectors $\left(B_{0}^{n}\right)^{k} \Phi$ are eigenvectors of the principal $\mathcal{L}_{n}$ number operator $B_{n-1}^{n-1}$ with eigenvalues $\left(\frac{\mu}{n}+k n(n-1)\right)$.

In agreement with Definition 1, for $k=0$ Definition 3 yields $B_{n-1}^{n-1} \Phi=\frac{\mu}{n} \Phi$.

3.2. Outline of the Fock space construction method. We will construct the TRHPWN Fock spaces by using the following method (cf. Chapter 3 of [18]):

(i) Compute

$$
\left\|\left(B_{0}^{n}\right)^{k} \Phi\right\|^{2}=\left\langle\left(B_{0}^{n}\right)^{k} \Phi,\left(B_{0}^{n}\right)^{k} \Phi\right\rangle=\pi_{n, k}(\mu)
$$

where $k=0,1,2, \ldots, \Phi$ is the $R H P W N$ vacuum vector, and $\pi_{n, k}(\mu)$ is a polynomial in $\mu$ of degree $k$.

(ii) Using the fact that if $k \neq m$ then $\left\langle\left(B_{0}^{n}\right)^{k} \Phi,\left(B_{0}^{n}\right)^{m} \Phi\right\rangle=0$, for $a, b \in \mathbb{C}$ compute

$$
\begin{aligned}
\left\langle e^{a B_{0}^{n}} \Phi, e^{b B_{0}^{n}} \Phi\right\rangle & =\sum_{k=0}^{\infty} \frac{(\bar{a} b)^{k}}{(k !)^{2}}\left\langle\left(B_{0}^{n}\right)^{k} \Phi,\left(B_{0}^{n}\right)^{k} \Phi\right\rangle \\
& =\sum_{k=0}^{\infty} \frac{(\bar{a} b)^{k}}{k !} \frac{\pi_{n, k}(\mu)}{k !}=\sum_{k=0}^{\infty} \frac{(\bar{a} b)^{k}}{k !} h_{n, k}(\mu)
\end{aligned}
$$

where

$$
h_{n, k}(\mu)=\frac{\pi_{n, k}(\mu)}{k !}
$$

(iii) Look for a function $G_{n}(u, \mu)$ such that

$$
G_{n}(u, \mu)=\sum_{k=0}^{\infty} \frac{u^{k}}{k !} h_{n, k}(\mu)
$$

Using the Taylor expansion of $G_{n}(u, \mu)$ in powers of $u$

$$
G_{n}(u, \mu)=\left.\sum_{k=0}^{\infty} \frac{u^{k}}{k !} \frac{\partial^{k}}{\partial u^{k}} G_{n}(u, \mu)\right|_{u=0}
$$

by comparing 3.7 and 3.6 we see that

$$
\left.\frac{\partial^{k}}{\partial u^{k}} G_{n}(u, \mu)\right|_{u=0}=h_{n, k}(\mu) .
$$

Equation (3.8) plays a fundamental role in the search for $G_{n}$ in what follows.

(iv) Reduce to single intervals and extend to step functions: For $u=\bar{a} b$, assuming that

$$
G_{n}(u, \mu)=e^{\mu \hat{G}_{n}(u)}
$$


which is typical for "Bernoulli moment systems" (cf. Chapter 5 of [18] ), equation 3.6 becomes

$$
e^{\mu \hat{G}_{n}(\bar{a} b)}=\sum_{k=0}^{\infty} \frac{(\bar{a} b)^{k}}{k !} h_{n, k}(\mu) .
$$

Take the product of 3.10 over all sets $I$, for test functions $f=\sum_{i} a_{i} \chi_{I_{i}}$ and $g=$ $\sum_{i} b_{i} \chi_{I_{i}}$ with $I_{i} \cap I_{j}=\oslash$ for $i \neq j$, and end up with an expression like

$$
e^{\int_{\mathbb{R}} \hat{G}_{n}(f(t) g(t)) d t}=\prod\left\langle e^{a B_{0}^{n}} \Phi, e^{b B_{0}^{n}} \Phi\right\rangle
$$

which we take as the definition of the inner product $\left\langle\psi_{n}(f), \psi_{n}(g)\right\rangle_{n}$ of the "exponential vectors"

$$
\psi_{n}(f)=\prod_{i} e^{a_{i} B_{0}^{n}\left(\chi_{I_{i}}\right)} \Phi
$$

of the $T R H P W N$ Fock space $\mathcal{F}_{n}$. Notice that $\Phi=\psi_{n}(0)$.

\subsection{Construction of the $T R H P W N$ Fock spaces $\mathcal{F}_{n}$}

Lemma 2. Let $n \geq 1$ be fixed. Then for all integers $k \geq 0$

$$
B_{n}^{0}\left(B_{0}^{n}\right)^{k+1} \Phi=n(k+1)\left(\mu+k \frac{n^{2}(n-1)}{2}\right)\left(B_{0}^{n}\right)^{k} \Phi .
$$

Proof. For $k=0$ we have

$$
\begin{aligned}
B_{n}^{0} B_{0}^{n} \Phi & =\left(B_{0}^{n} B_{n}^{0}+\left[B_{n}^{0}, B_{0}^{n}\right]\right) \Phi=0+n^{2} B_{n-1}^{n-1} \Phi \\
& =n^{2} \frac{\mu}{n} \Phi=n \mu \Phi=n(0+1)\left(\mu+0 \frac{n^{2}(n-1)}{2}\right)\left(B_{0}^{n}\right)^{0} \Phi .
\end{aligned}
$$

Assuming 3.13 to be true for $k$ we have

$$
\begin{aligned}
& B_{n}^{0}\left(B_{0}^{n}\right)^{k+2} \Phi=\left(B_{n}^{0} B_{0}^{n}\right)\left(B_{0}^{n}\right)^{k+1} \Phi=\left(B_{0}^{n} B_{n}^{0}+n^{2} B_{n-1}^{n-1}\right)\left(B_{0}^{n}\right)^{k+1} \Phi \\
& =B_{0}^{n} B_{n}^{0}\left(B_{0}^{n}\right)^{k+1} \Phi+n^{2} B_{n-1}^{n-1}\left(B_{0}^{n}\right)^{k+1} \Phi \\
& =B_{0}^{n} n(k+1)\left(\mu+k \frac{n^{2}(n-1)}{2}\right)\left(B_{0}^{n}\right)^{k} \Phi+n^{2} B_{n-1}^{n-1}\left(B_{0}^{n}\right)^{k+1} \Phi \\
& =\left(n(k+1)\left(\mu+k \frac{n^{2}(n-1)}{2}\right)+n^{2}\left(\frac{\mu}{n}+(k+1) n(n-1)\right)\right)\left(B_{0}^{n}\right)^{k+1} \Phi \\
& =n(k+2)\left(\mu+(k+1) \frac{n^{2}(n-1)}{2}\right)\left(B_{0}^{n}\right)^{k+1} \Phi,
\end{aligned}
$$

which proves 3.13 to be true for $k+1$ also, thus completing the induction.

Proposition 3. For all $n \geq 1$

$$
\pi_{n, k}(\mu)=\left\langle\left(B_{0}^{n}\right)^{k} \Phi,\left(B_{0}^{n}\right)^{k} \Phi\right\rangle=k ! n^{k} \prod_{i=0}^{k-1}\left(\mu+\frac{n^{2}(n-1)}{2} i\right) .
$$

Proof. Let $n \geq 1$ be fixed. Define

$$
a_{k}=k ! n^{k} \prod_{i=0}^{k-1}\left(\mu+\frac{n^{2}(n-1)}{2} i\right)
$$


Then $a_{1}=n \mu$ and for $k \geq 1$

$$
a_{k+1}=n(k+1)\left(\mu+k \frac{n^{2}(n-1)}{2}\right) a_{k} .
$$

Similarly, define

$$
b_{k}=\left\langle\left(B_{0}^{n}\right)^{k} \Phi,\left(B_{0}^{n}\right)^{k} \Phi\right\rangle .
$$

Then

$$
b_{1}=\left\langle B_{0}^{n} \Phi, B_{0}^{n} \Phi\right\rangle=\left\langle\Phi, B_{n}^{0} B_{0}^{n} \Phi\right\rangle=n^{2}\left\langle\Phi, B_{n-1}^{n-1} \Phi\right\rangle=n^{2} \frac{\mu}{n}=n \mu
$$

and for $k \geq 1$, using Lemma 2

$$
\begin{aligned}
b_{k+1} & =\left\langle\left(B_{0}^{n}\right)^{k} \Phi, B_{n}^{0}\left(B_{0}^{n}\right)^{k+1} \Phi\right\rangle=n(k+1)\left(\mu+k \frac{n^{2}(n-1)}{2}\right)\left\langle\left(B_{0}^{n}\right)^{k} \Phi,\left(B_{0}^{n}\right)^{k} \Phi\right\rangle \\
& =n(k+1)\left(\mu+k \frac{n^{2}(n-1)}{2}\right) b_{k} .
\end{aligned}
$$

Thus $a_{k}=b_{k}$ for all $k \geq 1$.

Corollary 1. The functions $h_{n, k}$ appearing in (3.5) are given by

$$
h_{1, k}=\mu^{k}
$$

and for $n \geq 2$

$$
h_{n, k}=n^{k} \prod_{i=0}^{k-1}\left(\mu+\frac{n^{2}(n-1)}{2} i\right) .
$$

Proof. The proof follows from Proposition 3 and (3.5).

Corollary 2. The functions $G_{n}$ appearing in (3.6) are given by

$$
G_{1}(u, \mu)=e^{u \mu}
$$

and for $n \geq 2$

$$
G_{n}(u, \mu)=\left(1-\frac{n^{3}(n-1)}{2} u\right)^{-\frac{2}{n^{2}(n-1)} \mu}=e^{-\frac{2}{n^{2}(n-1)} \mu \ln \left(1-\frac{n^{3}(n-1)}{2} u\right)}
$$

where ln denotes logarithm with base e.

Proof. The proof follows from the fact that for $G_{n}$ given by (3.17) and (3.18), in accordance with (3.8), we have

$$
\left.\frac{\partial^{k}}{\partial u^{k}} G_{n}(u, \mu)\right|_{u=0}=n^{k} \prod_{i=0}^{k-1}\left(\mu+\frac{n^{2}(n-1)}{2} i\right)
$$

COROLlary 3. The functions $\hat{G}_{n}$ appearing in (3.7) are given by

$$
\hat{G}_{1}(u)=u
$$

and for $n \geq 2$

$$
\hat{G}_{n}(u)=-\frac{2}{n^{2}(n-1)} \ln \left(1-\frac{n^{3}(n-1)}{2} u\right) .
$$

Proof. The proof follows directly from Corollary 2 . 
COROLlary 4. The $\mathcal{F}_{n}$ inner products are given by

$$
\left\langle\psi_{1}(f), \psi_{1}(g)\right\rangle_{1}=e^{\int_{\mathbb{R}} \bar{f}(t) g(t) d t}
$$

and for $n \geq 2$

$$
\left\langle\psi_{n}(f), \psi_{n}(g)\right\rangle_{n}=e^{-\frac{2}{n^{2}(n-1)} \int_{\mathbb{R}} \ln \left(1-\frac{n^{3}(n-1)}{2} \bar{f}(t) g(t)\right) d t}
$$

where $|f(t)|<\frac{1}{n} \sqrt{\frac{2}{n(n-1)}}$ and $|g(t)|<\frac{1}{n} \sqrt{\frac{2}{n(n-1)}}$.

Proof. The proof follows from 3.11 and Corollary 2

The function $G_{1}$ of 3.17 and the Fock space inner product (3.21) are associated with the Heisenberg algebra and the quantum stochastic calculus of 22 . For $n=2$ the function $G_{n}$ of $(3.18)$ and the associated Fock space inner product $(3.22)$ have appeared in the study of the Finite-Difference algebra and the Square of White Noise algebra in [12], [13, [16, and [17]. The functions $G_{n}$ of $(3.18$ can also be found in Proposition 5.4.2 of Chapter 5 of $[18$.

Definition 4. The $n$-th order TRHPWN Fock space $\mathcal{F}_{n}$ is the Hilbert space completion of the linear span of the exponential vectors $\psi_{n}(f)$ of $\left.\sqrt{3.12}\right)$ under the inner product $\langle\cdot, \cdot\rangle_{n}$ of Corollary 4. The full TRHPWN Fock space $\mathcal{F}$ is the direct sum of the $\mathcal{F}_{n}$ 's.

\subsection{Fock representation of the $T R H P W N$ operators}

Proposition 4. For all test functions $f=\sum_{i} a_{i} \chi_{I_{i}}$ and $g=\sum_{i} b_{i} \chi_{I_{i}}$ with $I_{i} \cap I_{j}=\oslash$ for $i \neq j$, and for all $n \geq 1$

$$
\begin{gathered}
B_{n}^{0}(f) \psi_{n}(g)=n \int_{\mathbb{R}} f(t) g(t) d t \psi_{n}(g)+\left.\frac{n^{3}(n-1)}{2} \frac{\partial}{\partial \epsilon}\right|_{\epsilon=0} \psi_{n}\left(g+\epsilon f g^{2}\right), \\
B_{0}^{n}(f) \psi_{n}(g)=\left.\frac{\partial}{\partial \epsilon}\right|_{\epsilon=0} \psi_{n}(g+\epsilon f) .
\end{gathered}
$$

Proof. By 3.12 , the fact that $\left[B_{n}^{0}\left(\chi_{I_{i}}\right), e^{B_{0}^{n}\left(\chi_{I_{j}}\right)}\right]=0$ whenever $I_{i} \cap I_{j}=\oslash$, and by Lemma 2 we have

$$
\begin{aligned}
B_{n}^{0}(f) \psi_{n}(g) & =\sum_{i=1}^{m} a_{i} B_{n}^{0}\left(\chi_{I_{i}}\right) \prod_{j=1}^{m} e^{b_{j} B_{0}^{n}\left(\chi_{I_{j}}\right)} \Phi \\
& =\sum_{i=1}^{m} a_{i} \prod_{j=1}^{m} B_{n}^{0}\left(\chi_{I_{i}}\right) e^{b_{j} B_{0}^{n}\left(\chi_{I_{j}}\right)} \Phi \\
& =\sum_{i=1}^{m} a_{i}\left(\prod_{\substack{j=1 \\
j \neq i}}^{m} e^{b_{j} B_{0}^{n}\left(\chi_{I_{j}}\right)}\right) B_{n}^{0}\left(\chi_{I_{i}}\right) e^{b_{i} B_{0}^{n}\left(\chi_{I_{i}}\right)} \Phi \\
& =\sum_{i=1}^{m} a_{i}\left(\prod_{\substack{j=1 \\
j \neq i}}^{m} e^{b_{j} B_{0}^{n}\left(\chi_{I_{j}}\right)}\right) \sum_{k=0}^{\infty} \frac{b_{i}^{k}}{k !} B_{n}^{0}\left(\chi_{I_{i}}\right)\left(B_{0}^{n}\left(\chi_{I_{i}}\right)\right)^{k} \Phi
\end{aligned}
$$




$$
\begin{aligned}
& =\sum_{i=1}^{m} a_{i}\left(\prod_{\substack{j=1 \\
j \neq i}}^{m} e^{b_{j} B_{0}^{n}\left(\chi_{I_{j}}\right)}\right) \sum_{k=0}^{\infty} \frac{b_{i}^{k}}{k !} n k\left(\mu\left(I_{i}\right)+(k-1) \frac{n^{2}(n-1)}{2}\right)\left(B_{0}^{n}\left(\chi_{I_{i}}\right)\right)^{k-1} \Phi \\
& =\sum_{i=1}^{m} a_{i}\left(\prod_{\substack{j=1 \\
j \neq i}}^{m} e^{b_{j} B_{0}^{n}\left(\chi_{I_{j}}\right)}\right) \sum_{k=1}^{\infty} \frac{b_{i}^{k}}{(k-1) !} n \mu\left(I_{i}\right)\left(B_{0}^{n}\left(\chi_{I_{i}}\right)\right)^{k-1} \Phi \\
& +\sum_{i=1}^{m} a_{i}\left(\prod_{\substack{j=1 \\
j \neq i}}^{m} e^{b_{j} B_{0}^{n}\left(\chi_{I_{j}}\right)}\right) \sum_{k=2}^{\infty} \frac{b_{i}^{k}}{(k-2) !} \frac{n^{3}(n-1)}{2}\left(B_{0}^{n}\left(\chi_{I_{i}}\right)\right)^{k-1} \Phi \\
& =n \sum_{i=1}^{m} a_{i} b_{i} \mu\left(I_{i}\right)\left(\prod_{\substack{j=1 \\
j \neq i}}^{m} e^{b_{j} B_{0}^{n}\left(\chi_{I_{j}}\right)}\right) e^{b_{i} B_{0}^{n}\left(\chi_{I_{i}}\right)} \Phi \\
& +\frac{n^{3}(n-1)}{2} \sum_{i=1}^{m} a_{i} b_{i}^{2} B_{0}^{n}\left(\chi_{I_{i}}\right)\left(\prod_{\substack{j=1 \\
j \neq i}}^{m} e^{b_{j} B_{0}^{n}\left(\chi_{I_{j}}\right)}\right) e^{b_{i} B_{0}^{n}\left(\chi_{I_{i}}\right)} \Phi \\
& =n \sum_{i=1}^{m} a_{i} b_{i} \mu\left(I_{i}\right)\left(\prod_{j=1}^{m} e^{b_{j} B_{0}^{n}\left(\chi_{I_{j}}\right)}\right) \Phi \\
& +\frac{n^{3}(n-1)}{2} \sum_{i=1}^{m} a_{i} b_{i}^{2} B_{0}^{n}\left(\chi_{I_{i}}\right) e^{b_{i} B_{0}^{n}\left(\chi_{I_{i}}\right)}\left(\prod_{\substack{j=1 \\
j \neq i}}^{m} e^{b_{j} B_{0}^{n}\left(\chi_{I_{j}}\right)}\right) \Phi \\
& =n \int_{\mathbb{R}} f(t) g(t) d t \psi_{n}(g)+\left.\frac{n^{3}(n-1)}{2} \sum_{i=1}^{m} \frac{\partial}{\partial \epsilon}\right|_{\epsilon=0} e^{\left(\epsilon a_{i} b_{i}^{2}+b_{i}\right) B_{0}^{n}\left(\chi_{I_{i}}\right)}\left(\prod_{\substack{j=1 \\
j \neq i}}^{m} e^{b_{j} B_{0}^{n}\left(\chi_{I_{j}}\right)}\right) \Phi \\
& =n \int_{\mathbb{R}} f(t) g(t) d t \psi_{n}(g)+\left.\frac{n^{3}(n-1)}{2} \frac{\partial}{\partial \epsilon}\right|_{\epsilon=0}\left(\prod_{i=1}^{m} e^{\left(\epsilon a_{i} b_{i}^{2}+b_{i}\right) B_{0}^{n}\left(\chi_{I_{i}}\right)}\right) \Phi \\
& =n \int_{\mathbb{R}} f(t) g(t) d t \psi_{n}(g)+\left.\frac{n^{3}(n-1)}{2} \frac{\partial}{\partial \epsilon}\right|_{\epsilon=0} \psi_{n}\left(g+\epsilon f g^{2}\right) \text {. }
\end{aligned}
$$

To prove 3.24 we notice that for $n=13.23$ yields

$$
B_{1}^{0}(f) \psi_{1}(g)=\int_{\mathbb{R}} f(t) g(t) d t \psi_{1}(g),
$$

i.e., $B_{1}^{0}(f)=A(f)$ where $A(f)$ is the annihilation operator of Hudson-Parthasarathy calculus (cf. [22]) and so

$$
B_{0}^{1}(f) \psi_{1}(g)=A^{\dagger}(f) \psi_{1}(g)=\left.\frac{\partial}{\partial \epsilon}\right|_{\epsilon=0} \psi_{1}(g+\epsilon f)
$$

where $A^{\dagger}(f)$ is the creation operator of Hudson-Parthasarathy calculus thus proving (3.24) for $n=1$. To prove (3.24) for $n \geq 2$ we notice that by the duality condition (1.4) for all test functions $f, g, \phi$

$$
\begin{aligned}
& \left\langle B_{0}^{n}(f) \psi_{n}(\phi), \psi_{n}(g)\right\rangle_{n}=\left\langle\psi_{n}(\phi), B_{n}^{0}(\bar{f}) \psi_{n}(g)\right\rangle_{n} \\
& \quad=n \int_{\mathbb{R}} \bar{f}(t) g(t) d t\left\langle\psi_{n}(\phi), \psi_{n}(g)\right\rangle_{n}+\left.\frac{n^{3}(n-1)}{2} \frac{\partial}{\partial \epsilon}\right|_{\epsilon=0}\left\langle\psi_{n}(\phi), \psi_{n}\left(g+\epsilon \bar{f} g^{2}\right)\right\rangle_{n}
\end{aligned}
$$




$$
\begin{aligned}
& =n \int_{\mathbb{R}} \bar{f}(t) g(t) d t\left\langle\psi_{n}(\phi), \psi_{n}(g)\right\rangle_{n} \\
& +\left.\frac{n^{3}(n-1)}{2} \frac{\partial}{\partial \epsilon}\right|_{\epsilon=0} e^{-\frac{2}{n^{2}(n-1)} \int_{\mathbb{R}} \ln \left(1-\frac{n^{3}(n-1)}{2} \bar{\phi}(t)\left(g+\epsilon \bar{f} g^{2}\right)(t)\right) d t} \\
& =n \int_{\mathbb{R}} \bar{f}(t) g(t) d t\left\langle\psi_{n}(\phi), \psi_{n}(g)\right\rangle_{n} \\
& +\frac{n^{3}(n-1)}{2}\left\langle\psi_{n}(\phi), \psi_{n}(g)\right\rangle_{n}\left(-\frac{2}{n^{2}(n-1)} \int_{\mathbb{R}} \frac{-\frac{n^{3}(n-1)}{1-\frac{n^{3}(n-1)}{2} \bar{\phi} \bar{f}} g^{2}}{\bar{\phi} g}(t) d t\right) \\
& =\left(n \int_{\mathbb{R}} \bar{f}(t) g(t) d t+\frac{n^{4}(n-1)}{2} \int_{\mathbb{R}} \frac{\bar{\phi}-\frac{n^{3}(n-1)}{2} \bar{\phi} g}{2}(t) d t\right)\left\langle\psi_{n}(\phi), \psi_{n}(g)\right\rangle_{n} \\
& =n \int_{\mathbb{R}} \frac{\bar{f} g}{1-\frac{n^{3}(n-1)}{2} \bar{\phi} g}(t) d t\left\langle\psi_{n}(\phi), \psi_{n}(g)\right\rangle_{n} \\
& =\left.\frac{\partial}{\partial \epsilon}\right|_{\epsilon=0} e^{-\frac{2}{n^{2}(n-1)} \int_{\mathbb{R}} \ln \left(1-\frac{n^{3}(n-1)}{2}(\bar{\phi}+\epsilon \bar{f})(t) g(t)\right) d t} \\
& =\left.\frac{\partial}{\partial \epsilon}\right|_{\epsilon=0} ^{\left\langle\psi_{n}(\phi+\epsilon f), \psi_{n}(g)\right\rangle_{n}} \\
& =\left\langle\left.\frac{\partial}{\partial \epsilon}\right|_{\epsilon=0} \psi_{n}(\phi+\epsilon f), \psi_{n}(g)\right\rangle_{n},
\end{aligned}
$$

which implies 3.24 .

Corollary 5. For all $n \geq 1$ and test functions $f, g, h$

$$
\begin{aligned}
& B_{n-1}^{n-1}(f g) \psi_{n}(h)=\frac{1}{n} \int_{\mathbb{R}} f(t) g(t) \psi_{n}(h) \\
& \quad+\left.\frac{n(n-1)}{2} \frac{\partial^{2}}{\partial \epsilon \partial \rho}\right|_{\epsilon=\rho=0}\left(\psi_{n}\left(h+\epsilon g+\rho f(h+\epsilon g)^{2}\right)-\psi_{n}\left(h+\epsilon f h^{2}+\rho g\right)\right) .
\end{aligned}
$$

Proof.

$$
\begin{aligned}
B_{n-1}^{n-1}(f g) \psi_{n}(h) & =\frac{1}{n^{2}}\left[B_{n}^{0}(f), B_{0}^{n}(g)\right] \psi_{n}(h) \\
& =\frac{1}{n^{2}}\left(B_{n}^{0}(f) B_{0}^{n}(g)-B_{0}^{n}(g) B_{n}^{0}(f)\right) \psi_{n}(h) \\
& =\frac{1}{n^{2}}\left(\left.B_{n}^{0}(f) \frac{\partial}{\partial \epsilon}\right|_{\epsilon=0} \psi_{n}(h+\epsilon g)-B_{0}^{n}(g)\left(n \int_{\mathbb{R}} f(t) h(t) d t \psi_{n}(h)\right.\right. \\
& \left.\left.+\left.\frac{n^{3}(n-1)}{2} \frac{\partial}{\partial \epsilon}\right|_{\epsilon=0} \psi_{n}\left(h+\epsilon f h^{2}\right)\right)\right) \\
& =\left.\frac{1}{n^{2}} \frac{\partial}{\partial \epsilon}\right|_{\epsilon=0} B_{n}^{0}(f) \psi_{n}(h+\epsilon g)-\frac{1}{n} \int_{\mathbb{R}} f(t) h(t) d t B_{0}^{n}(g) \psi_{n}(h) \\
& -\left.\frac{n(n-1)}{2} \frac{\partial}{\partial \epsilon}\right|_{\epsilon=0} B_{0}^{n}(g) \psi_{n}\left(h+\epsilon f h^{2}\right)
\end{aligned}
$$




$$
\begin{aligned}
& =\left.\frac{1}{n^{2}} \frac{\partial}{\partial \epsilon}\right|_{\epsilon=0}\left(n \int_{\mathbb{R}} f(t)(h+\epsilon g)(t) d t \psi_{n}(h+\epsilon g)\right. \\
& \left.+\left.\frac{n^{3}(n-1)}{2} \frac{\partial}{\partial \rho}\right|_{\rho=0} \psi_{n}\left(h+\epsilon g+\rho f(h+\epsilon g)^{2}\right)\right) \\
& -\left.\frac{1}{n} \int_{\mathbb{R}} f(t) h(t) d t \frac{\partial}{\partial \epsilon}\right|_{\epsilon=0} \psi_{n}(h+\epsilon g)-\left.\left.\frac{n(n-1)}{2} \frac{\partial}{\partial \epsilon}\right|_{\epsilon=0} \frac{\partial}{\partial \rho}\right|_{\rho=0} \psi_{n}\left(h+\epsilon f h^{2}+\rho g\right) \\
& =\frac{1}{n}\left(\int_{\mathbb{R}} f(t) g(t) d t \psi_{n}(h)+\left.\int_{\mathbb{R}} f(t) h(t) d t \frac{\partial}{\partial \epsilon}\right|_{\epsilon=0} \psi_{n}(h+\epsilon g)\right) \\
& +\left.\frac{n(n-1)}{2} \frac{\partial^{2}}{\partial \epsilon \partial \rho}\right|_{\epsilon=\rho=0} \psi_{n}\left(h+\epsilon g+\rho f(h+\epsilon g)^{2}\right) \\
& -\left.\frac{1}{n} \int_{\mathbb{R}} f(t) h(t) d t \frac{\partial}{\partial \epsilon}\right|_{\epsilon=0} \psi_{n}(h+\epsilon g) \\
& -\left.\frac{n(n-1)}{2} \frac{\partial^{2}}{\partial \epsilon \partial \rho}\right|_{\epsilon=\rho=0} \psi_{n}\left(h+\epsilon f h^{2}+\rho g\right) \\
& =\frac{1}{n} \int_{\mathbb{R}} f(t) g(t) d t \psi_{n}(h) \\
& +\left.\frac{n(n-1)}{2} \frac{\partial^{2}}{\partial \epsilon \partial \rho}\right|_{\epsilon=\rho=0}\left(\psi_{n}\left(h+\epsilon g+\rho f(h+\epsilon g)^{2}\right)-\psi_{n}\left(h+\epsilon f h^{2}+\rho g\right)\right) . \text { - }
\end{aligned}
$$

Using the method described in Corollary 5, i.e. using the prescription

$$
B_{k+K-1}^{n+N-1}(g f)=\frac{1}{k N-K n}\left(B_{k}^{n}(g) B_{K}^{N}(f)-B_{K}^{N}(f) B_{k}^{n}(g)\right)
$$

and suitable linear combinations, we obtain the representation of the $B_{y}^{x}$ (and therefore of the $R H P W N$ and Virasoro-Zamolodchikov- $w_{\infty}$ commutation relations) on the appropriate Fock space $\mathcal{F}_{n}$.

\section{Classical stochastic processes on $\mathcal{F}_{n}$}

Definition 5. A quantum stochastic process $x=\{x(t) / t \geq 0\}$ is a family of Hilbert space operators. Such a process is said to be classical if for all $t, s \geq 0, x(t)=x(t)^{*}$ and $[x(t), x(s)]=x(t) x(s)-x(s) x(t)=0$.

In the case of a Fock representation, the equation $x(t)=x(t)^{*}$ holds on the exponential domain (the linear span of the exponential vectors).

Proposition 5. Let $m>0$ and let a quantum stochastic process $x=\{x(t) / t \geq 0\}$ be defined by

$$
x(t)=\sum_{n, k \in \Lambda} c_{n, k} B_{k}^{n}(t)
$$

where $c_{n, k} \in \mathbb{C}-\{0\}, \Lambda$ is a finite subset of $\{0,1,2, \ldots\}$ and

$$
B_{k}^{n}(t)=B_{k}^{n}\left(\chi_{[0, t]}\right) \in \mathcal{F}_{m}
$$

If for each $n, k \in \Lambda$

$$
c_{n, k}=\bar{c}_{k, n}
$$

then the process $x=\{x(t) / t \geq 0\}$ is classical. 
Proof. By 1.4, $x(t)=x^{*}(t)$ for all $t \geq 0$. Moreover, by 1.6$],[x(t), x(s)]=0$ for all $t, s \geq 0$ since each term of the form $c_{N, K} c_{n, k}\left[B_{K}^{N}(t), B_{k}^{n}(s)\right]$ is canceled out by the corresponding term of the form $c_{n, k} c_{N, K}\left[B_{k}^{n}(s), B_{K}^{N}(t)\right]$. Thus the process $x=\{x(t) /$ $t \geq 0\}$ is classical.

In the remaining of this section we will study the classical process $x=\{x(t) / t \geq 0\}$ whose Fock representation as a family of operators on $\mathcal{F}_{n}$ is

$$
x(t)=B_{0}^{n}(t)+B_{n}^{0}(t) .
$$

By Proposition 4

$$
\begin{aligned}
& B_{0}^{n}(t) \psi_{n}(g)=\left.\frac{\partial}{\partial \epsilon}\right|_{\epsilon=0} \psi_{n}\left(g+\epsilon \chi_{[0, t]}\right), \\
& B_{n}^{0}(t) \psi_{n}(g)=n \int_{0}^{t} g(s) d s \psi_{n}(g)+\left.\frac{n^{3}(n-1)}{2} \frac{\partial}{\partial \epsilon}\right|_{\epsilon=0} \psi_{n}\left(g+\epsilon \chi_{[0, t]} g^{2}\right) .
\end{aligned}
$$

In particular, for $g=0$

$$
\begin{aligned}
& B_{0}^{n}(t) \psi_{n}(0)=\left.\frac{\partial}{\partial \epsilon}\right|_{\epsilon=0} \psi_{n}\left(\epsilon \chi_{[0, t]}\right), \\
& B_{n}^{0}(t) \psi_{n}(0)=0 .
\end{aligned}
$$

Lemma 3 (Splitting formula). Let $s \in \mathbb{R}$. Then for $n=1$

$$
e^{s\left(B_{0}^{1}+B_{1}^{0}\right)} \Phi=e^{\frac{s^{2}}{2} \mu} e^{s B_{0}^{1}} \Phi
$$

and for $n \geq 2$

$$
e^{s\left(B_{0}^{n}+B_{n}^{0}\right)} \Phi=\left(\sec \left(\sqrt{\frac{n^{3}(n-1)}{2}} s\right)\right)^{\frac{2 n \mu}{n^{3}(n-1)}} e^{\sqrt{\frac{2}{n^{3}(n-1)}} \tan \left(\sqrt{\frac{n^{3}(n-1)}{2}} s\right) B_{0}^{n}} \Phi .
$$

Proof. We will use the "differential method" of Proposition 4.1.1, Chapter 1 of [18]. So let

$$
E \Phi=e^{s\left(B_{0}^{n}+B_{n}^{0}\right)} \Phi=e^{V(s) B_{0}^{n}} e^{W(s)} \Phi
$$

where $W, V$ are real-valued functions with $W(0)=V(0)=0$. Then

$$
\frac{\partial}{\partial s} E \Phi=\left(B_{0}^{n}+B_{n}^{0}\right) E \Phi=B_{0}^{n} E \Phi+B_{n}^{0} E \Phi .
$$

By Lemma 2 we have

$$
\begin{aligned}
B_{n}^{0} E \Phi & =B_{n}^{0} e^{V(s) B_{0}^{n}} e^{W(s)} \Phi=e^{W(s)} B_{n}^{0} e^{V(s) B_{0}^{n} \Phi} \\
& =e^{W(s)} \sum_{k=0}^{\infty} \frac{V(s)^{k}}{k !} B_{n}^{0}\left(B_{0}^{n}\right)^{k} \Phi \\
& =e^{W(s)} \sum_{k=0}^{\infty} \frac{V(s)^{k}}{k !} n k\left(\mu+(k-1) \frac{n^{2}(n-1)}{2}\right)\left(B_{0}^{n}\right)^{k-1} \Phi \\
& =\left(n \mu V(s)+\frac{n^{3}(n-1)}{2} V(s)^{2} B_{0}^{n}\right) e^{V(s) B_{0}^{n}} e^{W(s)} \Phi \\
& =\left(n \mu V(s)+\frac{n^{3}(n-1)}{2} V(s)^{2} B_{0}^{n}\right) E \Phi .
\end{aligned}
$$


Thus 4.12 becomes

$$
\frac{\partial}{\partial s} E \Phi=\left(B_{0}^{n}+n \mu V(s)+\frac{n^{3}(n-1)}{2} V(s)^{2} B_{0}^{n}\right) E \Phi .
$$

From 4.11 we also have

$$
\frac{\partial}{\partial s} E \Phi=\left(V^{\prime}(s) B_{0}^{n}+W^{\prime}(s)\right) E \Phi .
$$

From 4.13 and 4.14, by equating coefficients of 1 and $B_{0}^{n}$, we have

$$
\begin{aligned}
W^{\prime}(s) & =n \mu V(s), \\
V^{\prime}(s) & =1+\frac{n^{3}(n-1)}{2} V(s)^{2} \text { (Riccati equation). }
\end{aligned}
$$

For $n=1$ we find $V(s)=s$ and $W(s)=\frac{s^{2}}{2} \mu$. For $n \geq 2$ by separating the variables we find

$$
V(s)=\sqrt{\frac{2}{n^{3}(n-1)}} \tan \left(\sqrt{\frac{n^{3}(n-1)}{2}} s\right)
$$

and so

$$
W(s)=-\frac{2 n \mu}{n^{3}(n-1)} \ln \left(\cos \left(\sqrt{\frac{n^{3}(n-1)}{2}} s\right)\right)
$$

which implies that

$$
e^{W(s)}=\left(\sec \left(\sqrt{\frac{n^{3}(n-1)}{2}} s\right)\right)^{\frac{2 n \mu}{n^{3}(n-1)}}
$$

thus completing the proof.

In the theory of Bernoulli systems and the Fock representation of finite-dimensional Lie algebras (cf. Chapter 5 of [18]) the Riccati equation (4.16) has the general form

$$
V^{\prime}(s)=1+2 \alpha V(s)+\beta V(s)^{2}
$$

and the values of $\alpha$ and $\beta$ determine the underlying classical probability distribution and the associated special functions. For example, for $\alpha=1-2 p$ and $\beta=-4 p q$ we have the binomial process and the Krawtchouk polynomials, for $\alpha=p^{-1}-\frac{1}{2}$ and $\beta=q p^{-2}$ we have the negative binomial process and the Meixner polynomials, for $\alpha \neq 0$ and $\beta=0$ we have the Poisson process and the Poisson-Charlier polynomials, for $\alpha^{2}=\beta$ we have the exponential process and the Laguerre polynomials, for $\alpha=\beta=0$ we have Brownian motion with moment generating function $e^{\frac{s^{2}}{2} t}$ and associated special functions the Hermite polynomials, and for $\alpha^{2}-\beta<0$ we have the continuous binomial and Beta processes (cf. Chapter 5 of [18] and also [20] ) with moment generating function $(\sec s)^{t}$ and associated special functions the Meixner-Pollaczek polynomials. In the infinite-dimensional TRHPWN case the underlying classical probability distributions are given in the following.

Proposition 6 (Moment generating functions). For all $s \geq 0$

$$
\left\langle e^{s\left(B_{0}^{1}(t)+B_{1}^{0}(t)\right)} \Phi, \Phi\right\rangle_{1}=e^{\frac{s^{2}}{2} t},
$$


i.e. $\left\{B_{0}^{1}(t)+B_{1}^{0}(t) / t \geq 0\right\}$ is Brownian motion (cf. [18], [22]) while for $n \geq 2$

$$
\left\langle e^{s\left(B_{0}^{n}(t)+B_{n}^{0}(t)\right)} \Phi, \Phi\right\rangle_{n}=\left(\sec \left(\sqrt{\frac{n^{3}(n-1)}{2}} s\right)\right)^{\frac{2 n t}{n^{3}(n-1)}},
$$

i.e. $\left\{B_{0}^{n}(t)+B_{n}^{0}(t) / t \geq 0\right\}$ is for each $n$ a continuous binomial/Beta process (see proposition 7 in the next section) corresponding to the density

$$
\mu_{t, n}(x)=p_{\frac{2 n}{n^{3}(n-1)}} t(x)=\frac{2^{\frac{2 n}{n^{3}(n-1)} t-1}}{2 \pi} B\left(\frac{\frac{2 n}{n^{3}(n-1)} t+i x}{2}, \frac{\frac{2 n}{n^{3}(n-1)} t-i x}{2}\right)
$$

where $B(a, c)$ is the Beta function with parameters a, $c$ as in 5.2) below.

Proof. The proof follows from Lemma $3, \mu([0, t])=t$, and the fact that for all $n \geq 1$ we have $B_{n}^{0}(t) \Phi=0$.

\section{The continuous binomial and Beta processes. Let}

$$
b_{n, k}(x)=\left(\begin{array}{l}
n \\
k
\end{array}\right) x^{k}(1-x)^{n-k}, \quad n, k \in\{0,1,2, \ldots\}, n \geq k, x \in(0,1),
$$

be the standard binomial distribution. Using the Gamma function we can analytically extend from $n, k \in\{0,1,2, \ldots\}$ to $z, w \in \mathbb{C}$ with $\Re z \geq \Re w>-1$ and we have

$$
\begin{aligned}
b_{z, w}(x) & =\frac{\Gamma(z+1)}{\Gamma(z-w+1) \Gamma(w+1)} x^{w}(1-x)^{z-w} \\
& =\frac{1}{z+1} \frac{\Gamma(z+2)}{\Gamma(z-w+1) \Gamma(w+1)} x^{w}(1-x)^{z-w} \\
& =\frac{1}{z+1} \frac{\Gamma(z+2)}{\Gamma(z-w+1) \Gamma(w+1)} x^{(w+1)-1}(1-x)^{(z-w+1)-1} \\
& =\frac{1}{z+1} \frac{1}{B(z-w+1, w+1)} x^{(w+1)-1}(1-x)^{(z-w+1)-1} \\
& =\frac{1}{z+1} \beta_{w+1, z-w+1}(x)
\end{aligned}
$$

where

$$
B(a, c)=\frac{\Gamma(a) \Gamma(c)}{\Gamma(a+c)}=\int_{0}^{1} x^{a-1}(1-x)^{c-1} d x, \quad \Re a>0, \Re c>0,
$$

is the Beta function and $\beta_{w+1, z-w+1}$ is the analytic continuation to $\Re a>0$ and $\Re c>0$ of the standard Beta distribution

$$
\beta_{a, c}(x)=\frac{\Gamma(a+c)}{\Gamma(a) \Gamma(c)} x^{a-1}(1-x)^{c-1}, \quad a>0, c>0 .
$$

Proposition 7. For each $t>0$ let $X_{t}$ be a random variable with distribution given by the density

$$
p_{t}(x)=\frac{2^{t-1}}{2 \pi} B\left(\frac{t+i x}{2}, \frac{t-i x}{2}\right)
$$

where $B$ is the Beta function. Then the moment generating function of $X_{t}$ is

$$
\left\langle e^{s X_{t}}\right\rangle=\int_{-\infty}^{\infty} e^{s x} p_{t}(x) d x=(\sec s)^{t}, \quad \forall t>0, s \in \mathbb{R} .
$$


Proof. See Proposition 4.1.1, Chapter 5 of [18].

Corollary 6. With $X_{t}$ and $p_{t}$ as in Proposition [7, let

$$
Y_{t}=\sqrt{\frac{n^{3}(n-1)}{2}} X_{t}
$$

Then the moment generating function of $Y_{t}$ with respect to the density

$$
q_{t}=p_{\frac{2 n}{n^{3}(n-1)}} t
$$

where $n \in\{1,2, \ldots\}$, is

$$
\left\langle e^{s Y_{t}}\right\rangle=\left(\sec \left(\sqrt{\frac{n^{3}(n-1)}{2}} s\right)\right)^{\frac{2 n t}{n^{3}(n-1)}} .
$$

Proof. Since $p_{t}$ is for each $t>0$ a probability density function we have

$$
\int_{-\infty}^{\infty} p_{t}(x) d x=1, \quad \forall t>0
$$

and so for $t:=\frac{2 n}{n^{3}(n-1)} t$

$$
\int_{-\infty}^{\infty} p_{\frac{2 n}{n^{3}(n-1)}} t(x) d x=1, \quad \forall t>0
$$

i.e.

$$
\int_{-\infty}^{\infty} q_{t}(x) d x=1, \quad \forall t>0,
$$

so $q_{t}$ is for each $t>0$ a probability density function. Moreover, letting $t:=\frac{2 n}{n^{3}(n-1)} t$ and $s:=\sqrt{\frac{n^{3}(n-1)}{2}} s$ in 5.5 we obtain

$$
\int_{-\infty}^{\infty} e^{s \sqrt{\frac{n^{3}(n-1)}{2}} x} q_{t}(x) d x=\left(\sec \left(\sqrt{\frac{n^{3}(n-1)}{2}} s\right)\right)^{\frac{2 n t}{n^{3}(n-1)}}
$$

which is precisely the moment generating function $\left\langle e^{s Y_{t}}\right\rangle$ of $Y_{t}$ with respect to $q_{t}$.

6. Central extensions of $R H P W N$ and $w_{\infty}$. The detailed proofs of all results presented in this section can be found in [6].

6.1. Basic concepts. If $L$ and $\widetilde{L}$ are two complex Lie algebras, we say that $\widetilde{L}$ is a one-dimensional central extension of $L$ with central element $E$ if there is a Lie algebra exact sequence $0 \rightarrow \mathbb{C} E \rightarrow \widetilde{L} \rightarrow L \rightarrow 0$, where $\mathbb{C} E$ is the one-dimensional trivial Lie algebra and the image of $\mathbb{C} E$ is contained in the center of $\widetilde{L}$. It is well-known that $\widetilde{L}$ is a one-dimensional central extension of $L$ if and only if $\widetilde{L}$ is the direct sum of $L$ and $\mathbb{C} E$ as vector spaces with

$$
\left[l_{1}, l_{2}\right]_{\widetilde{L}}=\left[l_{1}, l_{2}\right]_{L}+\phi\left(l_{1}, l_{2}\right) E
$$

and

$$
\left[l_{1}, E\right]_{\widetilde{L}}=0
$$

for all $l_{1}, l_{2} \in L$ where $[\cdot, \cdot]_{\widetilde{L}}$ and $[\cdot, \cdot]_{L}$ are the Lie brackets in $\widetilde{L}$ and $L$, respectively, and $\phi: L \times L \rightarrow \mathbb{C}$ is a bilinear (i.e. linear in both arguments) form on $L$ satisfying the 
skew-symmetry condition

$$
\phi\left(l_{1}, l_{2}\right)=-\phi\left(l_{2}, l_{1}\right)
$$

and the Jacobi identity

$$
\phi\left(\left[l_{1}, l_{2}\right]_{L}, l_{3}\right)+\phi\left(\left[l_{2}, l_{3}\right]_{L}, l_{1}\right)+\phi\left(\left[l_{3}, l_{1}\right]_{L}, l_{2}\right)=0 .
$$

In particular, 6.3 implies that $\phi(l, l)=0$ for all $l \in L$. The bilinear form $\phi$ is called a 2-cocycle on $L$. A central extension is trivial if $\widetilde{L}$ is the direct sum of a subalgebra $M$ and $\mathbb{C} E$ as Lie algebras, where the subalgebra $M$ is isomorphic to $L$. Two extensions are equivalent if each one of them is a trivial extension of the other, i.e. if their difference is a trivial cocycle. A 2-cocycle $\phi$ corresponding to a trivial central extension is called a 2-coboundary or a trivial 2-cocycle and it is given by a linear function $f: L \rightarrow \mathbb{C}$ satisfying

$$
\phi\left(l_{1}, l_{2}\right)=f\left(\left[l_{1}, l_{2}\right]_{L}\right)
$$

for all $l_{1}, l_{2} \in L$.

Notation 2. In the rest of this section we take all test functions to be the characteristic function of a fixed interval $I$ and we will from now on write $B_{k}^{n}$ instead of $B_{k}^{n}\left(\chi_{I}\right)$.

6.2. Central extensions of the Heisenberg algebra. The Heisenberg closed $*$-Lie subalgebra Heis of $R H P W N$ is generated by $B_{0}^{1}, B_{1}^{0}$ and $B_{0}^{0}$ with non-zero commutation relations

$$
\left[B_{1}^{0}, B_{0}^{1}\right]_{H e i s}=B_{0}^{0}
$$

and involution

$$
\left(B_{0}^{1}\right)^{*}=B_{1}^{0}, \quad\left(B_{1}^{0}\right)^{*}=B_{0}^{1}, \quad\left(B_{0}^{0}\right)^{*}=B_{0}^{0} .
$$

Proposition 8. (i) Let a 2-cocycle $\phi$ be defined on Heis $\times$ Heis through a bilinear skew-symmetric extension of

$$
\begin{aligned}
& \phi\left(B_{1}^{0}, B_{0}^{1}\right)=\lambda, \\
& \phi\left(B_{0}^{0}, B_{1}^{0}\right)=z, \\
& \phi\left(B_{0}^{1}, B_{0}^{0}\right)=\bar{z} \\
& \phi\left(B_{0}^{0}, B_{0}^{0}\right)=0 \\
& \phi\left(B_{0}^{1}, B_{0}^{1}\right)=0 \\
& \phi\left(B_{1}^{0}, B_{1}^{0}\right)=0
\end{aligned}
$$

where $\lambda \in \mathbb{R}$ and $z \in \mathbb{C}$. Then $\phi$ defines a central extension of Heis.

(ii) All 2-cocycles $\phi$ corresponding to a central extension of Heis are of the form (6.8)6.13 .

(iii) A central extension of Heis is trivial if and only if $z=0$.

In section 7 we discuss the non-trivial central extensions of the Heis $*$-Lie algebra in more detail. 
6.3. Central extensions of the Oscillator algebra. The Oscillator closed $*$-Lie subalgebra $O S C$ of $R H P W N$ is generated by $B_{0}^{1}, B_{1}^{0}, B_{0}^{0}$, and $B_{1}^{1}$ with non-zero commutation relations

$$
\begin{aligned}
& {\left[B_{1}^{0}, B_{0}^{1}\right]_{\text {OSC }}=B_{0}^{0},} \\
& {\left[B_{1}^{0}, B_{1}^{1}\right]_{\text {OSC }}=B_{1}^{0},} \\
& {\left[B_{1}^{1}, B_{0}^{1}\right]_{\text {OSC }}=B_{0}^{1},}
\end{aligned}
$$

and involution

$$
\left(B_{0}^{1}\right)^{*}=B_{1}^{0}, \quad\left(B_{1}^{0}\right)^{*}=B_{0}^{1}, \quad\left(B_{0}^{0}\right)^{*}=B_{0}^{0}, \quad\left(B_{1}^{1}\right)^{*}=B_{1}^{1} .
$$

Proposition 9. (i) Let a 2-cocycle $\phi$ be defined on $O S C \times O S C$ through a bilinear skew-symmetric extension of

$$
\begin{aligned}
& \phi\left(B_{1}^{0}, B_{0}^{1}\right)=\lambda, \\
& \phi\left(B_{0}^{0}, B_{0}^{1}\right)=0, \\
& \phi\left(B_{1}^{0}, B_{0}^{0}\right)=0, \\
& \phi\left(B_{1}^{0}, B_{1}^{1}\right)=z, \\
& \phi\left(B_{1}^{1}, B_{0}^{1}\right)=\bar{z}, \\
& \phi\left(B_{0}^{0}, B_{1}^{1}\right)=0,
\end{aligned}
$$

where $z \in \mathbb{C}, \lambda \in \mathbb{R}$. Then $\phi$ defines a central extension of $O S C$.

(ii) All 2-cocycles $\phi$ corresponding to a central extension of OSC are of the form (6.18)(6.23).

(iii) All central extensions of $O S C$ are trivial.

6.4. Central extensions of the Square of White Noise algebra. The Square of White Noise closed $*$-Lie subalgebra $S W N$ of $R H P W N$ is generated by $B_{0}^{2}, B_{2}^{0}$, and $B_{1}^{1}$ with non-zero commutation relations

$$
\begin{aligned}
& {\left[B_{2}^{0}, B_{1}^{1}\right]_{S W N}=2 B_{2}^{0},} \\
& {\left[B_{1}^{1}, B_{0}^{2}\right]_{S W N}=2 B_{0}^{2},} \\
& {\left[B_{2}^{0}, B_{0}^{2}\right]_{S W N}=4 B_{1}^{1},}
\end{aligned}
$$

and involution

$$
\left(B_{0}^{2}\right)^{*}=B_{2}^{0}, \quad\left(B_{2}^{0}\right)^{*}=B_{0}^{2}, \quad\left(B_{1}^{1}\right)^{*}=B_{1}^{1} .
$$

Proposition 10. (i) Let a 2-cocycle $\phi$ be defined on $S W N \times S W N$ through a bilinear skew-symmetric extension of

$$
\begin{aligned}
& \phi\left(B_{2}^{0}, B_{0}^{2}\right)=\lambda, \\
& \phi\left(B_{2}^{0}, B_{1}^{1}\right)=z, \\
& \phi\left(B_{1}^{1}, B_{0}^{2}\right)=\bar{z},
\end{aligned}
$$

where $z \in \mathbb{C}, \lambda \in \mathbb{R}$. Then $\phi$ defines a central extension of $S W N$.

(ii) All 2-cocycles $\phi$ corresponding to a central extension of $S W N$ are of the form (6.28)(6.30).

(iii) All central extensions of $S W N$ are trivial. 
6.5. Central extensions of $R H P W N$. Let $\overparen{R P P} N$ be a central extension of $R H P W N$ such that on the $R H P W N$ generators

$$
\begin{aligned}
{\left[B_{k}^{n}, B_{K}^{N}\right]_{R \widetilde{H P W} N} } & =\left[B_{k}^{n}, B_{K}^{N}\right]_{R H P W N}+c(n, k ; N, K) E \\
& =(k N-K n) B_{k+K-1}^{n+N-1}+c(n, k ; N, K) E
\end{aligned}
$$

with

$$
c(n, k ; N, K)=\phi\left(B_{k}^{n}, B_{K}^{N}\right) \in \mathbb{C}
$$

where $\phi$ is a cocycle as in 6.1.

Proposition 11. (i) For all nonnegative integers $n, k, N, K$ with $n+N=S+1$ and $k+K=M+1$, where $S, M \in\{-1,0,1,2, \ldots\}$ are given,

$$
c(n, k ; N, K)=z_{S, M} \cdot(k N-K n)
$$

where $z_{S, M} \in \mathbb{R}$.

(ii) All central extensions 6.31) of RHPWN are trivial.

6.6. Central extensions of $w_{\infty}$. Let $\widetilde{w_{\infty}}$ be a central extension of $w_{\infty}$ such that on the $w_{\infty}$ generators

$$
\begin{aligned}
{\left[\hat{B}_{k}^{n}, \hat{B}_{K}^{N}\right]_{\widetilde{w}_{\infty}} } & =\left[\hat{B}_{k}^{n}, \hat{B}_{K}^{N}\right]_{w_{\infty}}+c(n, k ; N, K) E \\
& =(k(N-1)-K(n-1)) \hat{B}_{k+K}^{n+N-2}+c(n, k ; N, K) E
\end{aligned}
$$

where $n, N \geq 2$ and $k, K \in \mathbb{Z}$, and

$$
c(n, k ; N, K)=\phi\left(\hat{B}_{k}^{n}, \hat{B}_{K}^{N}\right) \in \mathbb{C}
$$

where $\phi$ is a cocycle as in 6.1.

Proposition 12. (i) For all integers $n, k, N, K$ with $n, N \geq 2$ and $n+N=S+2$, $k+K=M$, where $S \geq 3$ (thus excluding the Witt-Virasoro sector $S=2$ ) and $M \in \mathbb{Z}$ are given,

$$
c(n, k ; N, K)=z_{S, M} \cdot(k(N-1)-K(n-1))
$$

where $z_{S, M} \in \mathbb{R}$.

(ii) All central extensions of $w_{\infty}$ are trivial.

The triviality of the central extensions of $w_{\infty}$ was first discussed in [14.

For the Witt-Virasoro sector of $w_{\infty}$ (corresponding to $S=2$ in proposition 12) we have the well-known non-trivial extension

$$
\left[\hat{B}_{m}^{2}, \hat{B}_{n}^{2}\right]_{w_{\infty}}=(m-n) \hat{B}_{m+n}^{2}+\delta_{m+n, 0} m\left(m^{2}-1\right) E .
$$

7. The centrally extended Heisenberg algebra. In this section (a continuation of subsection 6.2 above) we present our recently obtained results on the non-trivial central extensions of the Heisenberg algebra. Detailed proofs can be found in [7. For convenience we replace $B_{1}^{0}, B_{0}^{1}$ and $B_{0}^{0}$ by $a, a^{\dagger}$ and $h$ respectively and consider the (one mode) Heisenberg algebra Heis satisfying the Lie algebra commutation relations

$$
\left[a, a^{\dagger}\right]_{\text {Heis }}=h, \quad[a, h]_{\text {Heis }}=\left[h, a^{\dagger}\right]_{\text {Heis }}=0
$$


and the duality relations

$$
(a)^{*}=a^{\dagger}, \quad h^{\dagger}=h .
$$

As discussed in subsection 6.2, the Heisenberg algebra commutation relations can be centrally extended to

$$
\left[a, a^{\dagger}\right]_{C E H e i s}=h+\lambda E, \quad\left[h, a^{\dagger}\right]_{C E H e i s}=z E, \quad[a, h]_{C E H e i s}=\bar{z} E,
$$

where $E \not \equiv 0$ is the self-adjoint central element and where, here and in the following, all omitted commutators among generators are assumed to be equal to zero. The central extension is non-trivial if and only if $z \neq 0$.

Renaming $h+\lambda E$ by just $h$ in 7.3 we obtain the equivalent commutation relations

$$
\left[a, a^{\dagger}\right]_{C E H e i s}=h, \quad\left[h, a^{\dagger}\right]_{C E H e i s}=z E, \quad[a, h]_{C E H e i s}=\bar{z} E .
$$

From now on we will use (7.4) and 7.2 as the defining commutation relations and duality relations of the centrally extended Heisenberg algebra denoted by CEHeis. For $z=0$ we are back in the usual Heisenberg algebra.

For $\lambda \in \mathbb{R}$ and $z \in \mathbb{C} \backslash\{0\}$ commutation relations (7.4) define a solvable fourdimensional $*$-Lie algebra $C E H$ eis with generators $a, a^{\dagger}, h$ and $E$. Defining $p, q$ and $H$ by

$$
a^{\dagger}=p+i q, \quad a=p-i q, \quad H=-i h / 2,
$$

we have that $p, q, E$ are self-adjoint and $H$ is skew-adjoint. Moreover $p, q, E$ and $H$ are the generators of a real four-dimensional solvable *-Lie algebra RCEHeis with central element $E$ and commutation relations

$$
[p, q]=H, \quad[q, H]=c E, \quad[H, p]=b E,
$$

where $b, c$ are (not simultaneously zero) real numbers given by

$$
c=\frac{\operatorname{Re} z}{2}, \quad b=\frac{\operatorname{Im} z}{2} .
$$

Conversely, if $p, q, H, E$ are the generators (with $p, q, E$ self-adjoint and $H$ skew-adjoint) of a real four-dimensional solvable $*$-Lie algebra with central element $E$ and commutation relations (7.6) where $b$ and $c$ are (not simultaneously zero) real numbers then, defining $z$ by 7.7 , the operators defined by $(7.5)$ are the generators of the nontrivial central extension $C E H e i s$ of the Heisenberg algebra defined by (7.4), 7.2).

Real four-dimensional solvable Lie algebras are fully classified. There are exactly fifteen isomorphism classes and they are listed, for example, in proposition 2.1 of [25] (see references therein for additional information). One of the fifteen Lie algebras that appear in the above mentioned classification list is the Lie algebra $\eta_{4}$ with generators $\xi_{1}, \xi_{2}, \xi_{3}, \xi_{4}$ and (non-zero) commutation relations among generators

$$
\left[\xi_{4}, \xi_{1}\right]=\xi_{2}, \quad\left[\xi_{4}, \xi_{2}\right]=\xi_{3} .
$$

The real four-dimensional solvable Lie algebra RCEHeis described above can be identified to $\eta_{4}$. The Lie algebra $\eta_{4}$ and the corresponding Lie group have also been studied by Feinsilver and Schott in [19]. But it is the first time that its connection with the non-trivial central extensions of an important Lie algebra such as the Heisenberg one has been pointed out. 
The generators $a, a^{\dagger}, h$ and $E$ of $C E H$ eis can be expressed in terms of the generators $b, b^{\dagger}, b^{2}, b^{\dagger^{2}}, b^{\dagger} b$ and 1 of the Schrödinger algebra. Here $b^{\dagger}, b$ and 1 are the generators of a Boson Heisenberg algebra with

$$
\left[b, b^{\dagger}\right]=1, \quad\left(b^{\dagger}\right)^{*}=b .
$$

More precisely

(i) If $z \in \mathbb{C}$ with $\operatorname{Re} z \neq 0$ then for arbitrary $\rho, r \in \mathbb{R}$ with $r \neq 0$ the quadruple $\left\{a^{+}, a, h, E=1\right\}$ where:

$$
\begin{aligned}
a & =\left(\frac{4 \rho \operatorname{Im} z-r^{2}}{4 \operatorname{Re} z}+i \rho\right)\left(b-b^{\dagger}\right)^{2}-\frac{i \bar{z}}{2 r}\left(b+b^{\dagger}\right), \\
a^{\dagger} & =\left(\frac{4 \rho \operatorname{Im} z-r^{2}}{4 \operatorname{Re} z}-i \rho\right)\left(b-b^{\dagger}\right)^{2}+\frac{i z}{2 r}\left(b+b^{\dagger}\right),
\end{aligned}
$$

and

$$
h=i r\left(b^{\dagger}-b\right)
$$

satisfies the commutation relations (7.4) and the duality relations $(7.2)$ of CEHeis.

(ii) If $z \in \mathbb{C}$ with $R e z=0$ then for arbitrary $\rho, r \in \mathbb{R}$ with $r \neq 0$ the quadruple $\left\{a^{+}, a, h, E=1\right\}$ where:

$$
\begin{aligned}
a & =\left(\rho-\frac{i \operatorname{Im} z}{16 r^{2}}\right)\left(b-b^{\dagger}\right)^{2}+r\left(b+b^{\dagger}\right), \\
a^{\dagger} & =\left(\rho+\frac{i \operatorname{Im} z}{16 r^{2}}\right)\left(b-b^{\dagger}\right)^{2}+r\left(b+b^{\dagger}\right),
\end{aligned}
$$

and

$$
h=\frac{i \operatorname{Im} z}{2 r}\left(b^{\dagger}-b\right),
$$

satisfies the commutation relations (7.4) and the duality relations 7.2 of $C E$ Heis.

We can therefore represent the generators $a, a^{\dagger}, h$ and $E$ of $C E H e i s$ on the usual Heisenberg Fock space (with vacuum vector $\Phi$ such that $b \Phi=0$ and $\|\Phi\|=1$ ) defined as the Hilbert space completion of the linear span of the exponential vectors $\{y(\lambda)=$ $\left.e^{\lambda b^{\dagger}} \Phi ; \lambda \in \mathbb{C}\right\}$ with respect to the inner product

$$
\langle y(\lambda), y(\mu)\rangle=e^{\bar{\lambda} \mu}
$$

using the well-known representation, for non-negative integers $n$ and $k$,

$$
b^{\dagger^{n}} b^{k} y(\lambda)=\left.\lambda^{k} \frac{\partial^{n}}{\partial \epsilon^{n}}\right|_{\epsilon=0} y(\lambda+\epsilon) .
$$

In fact:

(i) If $z \in \mathbb{C}$ with $\operatorname{Re} z \neq 0$ then 


$$
\begin{gathered}
a y(\lambda)=\left(\left(\frac{4 \rho \operatorname{Im} z-r^{2}}{4 \operatorname{Re} z}+i \rho\right)\left(\lambda^{2}-1\right)-\frac{i \bar{z}}{2 r} \lambda\right) y(\lambda) \\
+\left(\left.\left(\frac{4 \rho \operatorname{Im} z-r^{2}}{4 \operatorname{Re} z}+i \rho\right) \frac{\partial^{2}}{\partial \epsilon^{2}}\right|_{\epsilon=0}\right. \\
\left.-\left.\left(\left(\frac{4 \rho \operatorname{Im} z-r^{2}}{4 \operatorname{Re} z}+i \rho\right) 2 \lambda+\frac{i \bar{z}}{2 r}\right) \frac{\partial}{\partial \epsilon}\right|_{\epsilon=0}\right) y(\lambda+\epsilon), \\
a^{\dagger} y(\lambda)=\left(\left(\frac{4 \rho \operatorname{Im} z-r^{2}}{4 \operatorname{Re} z}-i \rho\right)\left(\lambda^{2}-1\right)+\frac{i z}{2 r} \lambda\right) y(\lambda) \\
+\left(\left.\left(\frac{4 \rho \operatorname{Im} z-r^{2}}{4 \operatorname{Re} z}-i \rho\right) \frac{\partial^{2}}{\partial \epsilon^{2}}\right|_{\epsilon=0}\right. \\
\left.-\left.\left(\left(\frac{4 \rho \operatorname{Im} z-r^{2}}{4 \operatorname{Re} z}-i \rho\right) 2 \lambda-\frac{i z}{2 r}\right) \frac{\partial}{\partial \epsilon}\right|_{\epsilon=0}\right) y(\lambda+\epsilon), \\
h y(\lambda)=i r\left(\left.\frac{\partial}{\partial \epsilon}\right|_{\epsilon=0} y(\lambda+\epsilon)-\lambda y(\lambda)\right),
\end{gathered}
$$

and

$$
E y(\lambda)=y(\lambda)
$$

(ii) If $z \in \mathbb{C}$ with $\operatorname{Re} z=0$ then

$$
\begin{aligned}
& a y(\lambda)=\left(\left(\rho-\frac{i \operatorname{Im} z}{16 r^{2}}\right)\left(\lambda^{2}-1\right)+r \lambda\right) y(\lambda) \\
&+\left(\left.\left(\rho-\frac{i \operatorname{Im} z}{16 r^{2}}\right) \frac{\partial^{2}}{\partial \epsilon^{2}}\right|_{\epsilon=0}+\left.\left(r-\left(\rho-\frac{i \operatorname{Im} z}{16 r^{2}}\right) 2 \lambda\right) \frac{\partial}{\partial \epsilon}\right|_{\epsilon=0}\right) y(\lambda+\epsilon), \\
& a^{\dagger} y(\lambda)=\left(\left(\rho+\frac{i \operatorname{Im} z}{16 r^{2}}\right)\left(\lambda^{2}-1\right)+r \lambda\right) y(\lambda) \\
&+\left(\left.\left(\rho+\frac{i \operatorname{Im} z}{16 r^{2}}\right) \frac{\partial^{2}}{\partial \epsilon^{2}}\right|_{\epsilon=0}+\left.\left(r-\left(\rho+\frac{i \operatorname{Im} z}{16 r^{2}}\right) 2 \lambda\right) \frac{\partial}{\partial \epsilon}\right|_{\epsilon=0}\right) y(\lambda+\epsilon), \\
& h y(\lambda)=\frac{i \operatorname{Im} z}{2 r}\left(\left.\frac{\partial}{\partial \epsilon}\right|_{\epsilon=0} y(\lambda+\epsilon)-\lambda y(\lambda)\right),
\end{aligned}
$$

and

$$
E y(\lambda)=y(\lambda)
$$

REMARK 1. CEHeis can also be represented in terms of two independent CCR copies. In fact, for $j, k \in\{1,2\}$ letting $\left[q_{j}, p_{k}\right]=\frac{i}{2} \delta_{j, k}$ and $\left[q_{j}, q_{k}\right]=\left[p_{j}, p_{k}\right]=0$ with $p_{j}^{\dagger}=p_{j}$, $q_{j}^{\dagger}=q_{j}$ and $i^{2}=-1$, we have:

(i) If $z \in \mathbb{C}$ with $\operatorname{Re} z \neq 0$ and $\operatorname{Im} z \neq 0$ then

$$
\begin{aligned}
a & =i \operatorname{Re} z q_{1}+\frac{1}{\operatorname{Re} z} p_{1}^{2}-\operatorname{Im} z p_{2}-\frac{i}{\operatorname{Im} z} q_{2}^{2}, \\
a^{\dagger} & =-i \operatorname{Re} z q_{1}+\frac{1}{\operatorname{Re} z} p_{1}^{2}-\operatorname{Im} z p_{2}+\frac{i}{\operatorname{Im} z} q_{2}^{2}, \\
h & =-2\left(p_{1}+q_{2}\right),
\end{aligned}
$$


and $E=1$ satisfy the commutation relations $(7.4)$ and the duality relations 7.2 of CEHeis.

(ii) If $z \in \mathbb{C}$ with $\operatorname{Re} z=0$ and $\operatorname{Im} z \neq 0$ then for arbitrary $r \in \mathbb{R}$ and $c \in \mathbb{C}$

$$
\begin{aligned}
a & =c p_{1}^{2}-\operatorname{Im} z p_{2}+\left(r-\frac{i}{\operatorname{Im} z}\right) q_{2}^{2}, \\
a^{\dagger} & =\bar{c} p_{1}^{2}-\operatorname{Im} z p_{2}+\left(r+\frac{i}{\operatorname{Imz}}\right) q_{2}^{2}, \\
h & =-2 q_{2},
\end{aligned}
$$

and $E=1$ satisfy the commutation relations 7.4 and the duality relations 7.2 of CEHeis.

(iii) If $z \in \mathbb{C}$ with $\operatorname{Re} z \neq 0$ and $\operatorname{Im} z=0$ then for arbitrary $r \in \mathbb{R}$ and $c \in \mathbb{C}$

$$
\begin{aligned}
a & =i \operatorname{Re} z q_{1}+\left(\frac{1}{\operatorname{Re} z}+i r\right) p_{1}^{2}+c q_{2}^{2}, \\
a^{\dagger} & =-i \operatorname{Re} z q_{1}+\left(\frac{1}{\operatorname{Re} z}-i r\right) p_{1}^{2}+\bar{c} q_{2}^{2}, \\
h & =-2 p_{1},
\end{aligned}
$$

and $E=1$ satisfy the commutation relations $(7.4)$ and the duality relations 7.2 of CEHeis.

8. Random variables in CEHeis. Self-adjoint operators $X$ on the Heisenberg Fock space correspond to classical random variables with moment generating function $\left\langle\Phi, e^{s X} \Phi\right\rangle$ where $s \in \mathbb{R}$. In this section we compute the moment generating function of the selfadjoint operator $X=a+a^{\dagger}+h$. All proofs can be found in [7].

Lemma 4 (Splitting formula). Let $L \in \mathbb{R}$ and $M, N \in \mathbb{C}$. Then for all $s \in \mathbb{R}$ such that $2 L s+1>0$

$$
e^{s\left(L b^{2}+L b^{\dagger^{2}}-2 L b^{\dagger} b-L+M b+N b^{\dagger}\right)} \Phi=e^{w_{1}(s) b^{\dagger^{2}}} e^{w_{2}(s) b^{\dagger}} e^{w_{3}(s)} \Phi
$$

where

$$
\begin{aligned}
w_{1}(s) & =\frac{L s}{2 L s+1}, \\
w_{2}(s) & =\frac{L(M+N) s^{2}+N s}{2 L s+1},
\end{aligned}
$$

and

$$
w_{3}(s)=\frac{(M+N)^{2}\left(L^{2} s^{4}+2 L s^{3}\right)+3 M N s^{2}}{6(2 L s+1)}-\frac{\ln (2 L s+1)}{2}
$$

are respectively the solutions of

$$
\begin{aligned}
& w_{1}^{\prime}(s)=4 L w_{1}(s)^{2}-4 L w_{1}(s)+L \text { (Riccati differential equation), } \\
& w_{2}^{\prime}(s)=\left(4 L w_{1}(s)-2 L\right) w_{2}(s)+2 M w_{1}(s)+N \text { (Linear differential equation), } \\
& w_{3}^{\prime}(s)=2 L w_{1}(s)+L w_{2}(s)^{2}-L+M w_{2}(s),
\end{aligned}
$$

with $w_{1}(0)=w_{2}(0)=w_{3}(0)=0$. 
REMARK 2. For $L \neq 0$ the Riccati equation

$$
w_{1}^{\prime}(s)=4 L w_{1}(s)^{2}-4 L w_{1}(s)+L
$$

can be put in the canonical form

$$
V^{\prime}(s)=1+2 \alpha V(s)+\beta V(s)^{2}
$$

of the theory of Bernoulli systems of chapters 5 and 6 of [18], where $V(s)=w_{1}(s) / L$, $\alpha=-2 L$ and $\beta=4 L^{2}$. Then $\delta^{2}:=\alpha^{2}-\beta=0$ which is characteristic of exponential and Gaussian systems ([18, Proposition 5.3.2). For $L=0$ we obtain classical Brownian motion (see proposition 13 below).

Proposition 13 (Moment Generating Function). For all $s \in \mathbb{R}$ such that 2 L $s+1>0$

$$
\left\langle\Phi, e^{s\left(a+a^{\dagger}+h\right)} \Phi\right\rangle=(2 L s+1)^{-1 / 2} e^{\frac{(M+N)^{2}\left(L^{2} s^{4}+2 L s^{3}\right)+3 M N s^{2}}{6(2 L s+1)}}
$$

where in the notation of 7.10 - 7.15

(i) if $\operatorname{Re} z \neq 0$ then

$$
\begin{aligned}
L & =\frac{4 \rho \operatorname{Im} z-r^{2}}{2 \operatorname{Re} z}, \\
M & =-\left(\frac{\operatorname{Im} z}{r}+i r\right), \\
N & =-\left(\frac{\operatorname{Im} z}{r}-i r\right),
\end{aligned}
$$

(ii) if $\operatorname{Re} z=0$ then

$$
\begin{aligned}
L & =2 \rho, \\
M & =2 r-i \frac{\operatorname{Im} z}{2 r}, \\
N & =2 r+i \frac{\operatorname{Im} z}{2 r} .
\end{aligned}
$$

REMARK 3. If $L=0$ (corresponding to $\rho \operatorname{Im} z>0$ and $r^{2}=4 \rho \operatorname{Im} z$ in the case when $\operatorname{Re} z \neq 0$ and to $\rho=0$ in the case when $\operatorname{Re} z=0)$ then 8.10 becomes

$$
\left\langle\Phi, e^{s\left(a+a^{\dagger}+h\right)} \Phi\right\rangle=e^{\frac{M N s^{2}}{2}}= \begin{cases}e^{\left(\frac{(I m z)^{2}}{2 r^{2}}+\frac{r^{2}}{2}\right) s^{2}} & \text { if } \operatorname{Re} z \neq 0, \\ e^{\left(2 r^{2}+\frac{(I m z)^{2}}{8 r^{2}}\right) s^{2}} & \text { if } \operatorname{Re} z=0,\end{cases}
$$

which means that $a+a^{\dagger}+h$ is a Gaussian random variable.

For $L \neq 0$ the term $(2 L s+1)^{-1 / 2}$ appearing in 8.10 is the moment generating function of a gamma random variable.

9. The centrally extended Heisenberg Lie group. By exponentiating the elements of $C E H e i s$ we obtain the elements of the centrally extended Heisenberg Lie group. In this section we provide the group law. All proofs can be found in [7].

Lemma 5. For all $X, Y \in \operatorname{span}\left\{a, a^{\dagger}, h, E\right\}$

$$
e^{X+Y}=e^{X} e^{Y} e^{-\frac{1}{2}[X, Y]} e^{\frac{1}{6}(2[Y,[X, Y]]+[X,[X, Y]])} .
$$


Lemma 6. For all $\lambda, \mu \in \mathbb{C}$

$$
\begin{gathered}
e^{\lambda a} e^{\mu a^{\dagger}}=e^{\mu a^{\dagger}} e^{\lambda a} e^{\lambda \mu h} e^{\frac{\lambda \mu}{2}(\mu z-\lambda \bar{z}),} \\
a e^{\mu a^{\dagger}}=e^{\mu a^{\dagger}}\left(a+\mu h+\frac{\mu^{2} z}{2}\right), \\
e^{\lambda a} e^{\mu h}=e^{\mu h} e^{\lambda a} e^{\lambda \mu \bar{z}}, \\
e^{\mu h} e^{\lambda a^{\dagger}}=e^{\lambda a^{\dagger}} e^{\mu h} e^{\lambda \mu z}, \\
a e^{\mu h}=e^{\mu h}(a+\mu \bar{z}), \\
h e^{\lambda a^{\dagger}}=e^{\lambda a^{\dagger}}(h+\lambda z) .
\end{gathered}
$$

Proposition 14 (Group Law). For $u, v, w, y \in \mathbb{C}$ define

$$
g(u, v, w, y)=e^{u a^{\dagger}} e^{v h} e^{w a} e^{y E} .
$$

Then the family of operators of the form (9.8) is a group with group law given by

$$
\begin{gathered}
g(\alpha, \beta, \gamma, \delta) g(A, B, C, D) \\
=g\left(\alpha+A, \beta+B+\gamma A, \gamma+C,\left(\frac{\gamma A^{2}}{2}+\beta A\right) z+\left(\frac{\gamma^{2} A}{2}+\gamma B\right) \bar{z}+\delta+D\right) .
\end{gathered}
$$

The family of operators of the form 9.8 with $u, v, w \in \mathbb{R}$ and $y \in \mathbb{C}$ is a sub-group. The group $\mathbb{R}^{3} \times \mathbb{C}$ endowed with the composition law:

$$
\begin{gathered}
(\alpha, \beta, \gamma, \delta)(A, B, C, D) \\
=\left(\alpha+A, \beta+B+\gamma A, \gamma+C,\left(\frac{\gamma A^{2}}{2}+\beta A\right) z+\left(\frac{\gamma^{2} A}{2}+\gamma B\right) \bar{z}+\delta+D\right)
\end{gathered}
$$

is called the centrally extended Heisenberg group.

10. Matrix representation of CEHeis. In example (ix) of [19], Feinsilver and Schott considered the Lie algebra $\eta_{4}$ generated by $\left\{\xi_{i} / i=1,2,3,4\right\}$ where

$$
\left[\xi_{4}, \xi_{1}\right]=\xi_{2} ;\left[\xi_{4}, \xi_{2}\right]=\xi_{3}
$$

and all other commutators (between the generators) are equal to zero. This is precisely the Lie algebra $\eta_{4}$ mentioned in section 7 above.

REMARK 4. As mentioned in [19], the generators $\xi_{1}, \xi_{2}, \xi_{3}, \xi_{4}$ can be represented on the space of smooth functions $f(x)$ as $x^{2} / 2, x, 1$ and $D=d / d x$ respectively, with $[D, x]=1$. However, this representation does not allow us to view $\eta_{4}$ as a $*$-Lie algebra since by taking the adjoint of commutation relations [10.1] (this is the "left dual" of [19] denoted by $\dagger$ ) we have that

$$
\left[D, x^{2} / 2\right]^{\dagger}=x^{\dagger}, \quad[D, x]^{\dagger}=1^{\dagger}
$$

i.e.

$$
\left[D^{2} / 2, x\right]=D ;[D, x]=1,
$$

and so, with this representation, $\eta_{4}$ is not closed under taking adjoints. 
A matrix representation of $\eta_{4}$ was given by Feinsilver and Schott to be

$$
\sum_{i=1}^{4} \alpha_{i} \xi_{i}=\left[\begin{array}{cccc}
0 & \alpha_{4} & 0 & \alpha_{3} \\
0 & 0 & \alpha_{4} & \alpha_{2} \\
0 & 0 & 0 & \alpha_{1} \\
0 & 0 & 0 & 0
\end{array}\right]
$$

where $\alpha_{i} \in \mathbb{C}$ for $i=1,2,3,4$ and

$$
e^{\sum_{i=1}^{4} \alpha_{i} \xi_{i}}=\left[\begin{array}{cccc}
1 & \alpha_{4} & \frac{\alpha_{4}^{2}}{2} & \frac{\alpha_{4}^{2} \alpha_{1}}{6}+\frac{\alpha_{4} \alpha_{2}}{2}+\alpha_{3} \\
0 & 1 & \alpha_{4} & \frac{\alpha_{4} \alpha_{1}}{2}+\alpha_{2} \\
0 & 0 & 1 & \alpha_{1} \\
0 & 0 & 0 & 1
\end{array}\right]
$$

Defining group elements $g(a, b, c, d)=e^{a \xi_{1}} e^{b \xi_{2}} e^{c \xi_{3}} e^{d \xi_{4}}$ through matrix multiplication they verified the group law

$$
g(a, b, c, d) g(A, B, C, D)=g\left(a+A, b+B+d A, c+C+d B+\frac{1}{2} d^{2} A, d+D\right)
$$

since both sides are equal to

$$
\left[\begin{array}{cccc}
1 & d+D & \frac{d^{2}}{2}+d D+\frac{D^{2}}{2} & c+\frac{d^{2} A}{2}+d B+C \\
0 & 1 & d+D & b+d A+B \\
0 & 0 & 1 & a+A \\
0 & 0 & 0 & 1
\end{array}\right]
$$

To connect $\eta_{4}$ with $C E H e i s$ we let

$$
z=2 c+2 b i
$$

where $b$ and $c$ are not simultaneously zero and we distinguish the following cases:

Case (i). $b=0$ and $c \neq 0$ : Take $q=\xi_{4}, p=\xi_{1}, H=-\xi_{2}$ and $E=-\frac{1}{c} \xi_{3}$ and define $a=p-i q, a^{\dagger}=p+i q$ and $h=2 i H$.

Case (ii). $b \neq 0$ and $c=0$ : Take $p=\xi_{4}, q=\xi_{1}, H=\xi_{2}$ and $E=-\frac{1}{b} \xi_{3}$ and define $a=p-i q, a^{\dagger}=p+i q$ and $h=2 i H$.

Case (iii). $b \neq 0$ and $c \neq 0$ : Take $p=\beta \xi_{4}, q=\frac{1}{\beta} \xi_{1}-\alpha \xi_{4}, H=\xi_{2}$ and $E=-\frac{\beta}{b} \xi_{3}$, where $\alpha, \beta \in \mathbb{R} \backslash\{0\}$ are such that $\beta c-\alpha b=0$, and define $a=p-i q, a^{\dagger}=p+i q$ and $h=2 i H$.

In all cases (i)-(iii) $p, q, H$ and $E$ satisfy commutation relations 7.6 and $a^{+}, a, h$ and $E$ satisfy the commutation relations 7.4 of $C E H e i s$. If we introduce the duality relations

$$
\xi_{1}^{*}=\xi_{1} ; \xi_{2}^{*}=-\xi_{2} ; \xi_{3}^{*}=\xi_{3} ; \xi_{4}^{*}=\xi_{4}
$$

we conclude that $a^{+}, a, h$ and $E$ also satisfy the duality relations 7.2 of CEHeis. 
Using (10.4) we can obtain a matrix representation of $C$ EHeis (satisfying commutation relations (7.4) but not duality relations (7.2)) in each of the cases (i)-(iii) described above.

Case (i). $z=2 c$ where $c \in \mathbb{R} \backslash\{0\}$ :

$$
\begin{gathered}
\alpha_{1} a+\alpha_{2} a^{\dagger}+\alpha_{3} h+\alpha_{4} E=\left(\alpha_{1}+\alpha_{2}\right) \xi_{1}-2 i \alpha_{3} \xi_{2}-\frac{1}{c} \alpha_{4} \xi_{3}+i\left(\alpha_{2}-\alpha_{1}\right) \xi_{4} \\
=\left[\begin{array}{cccc}
0 & i\left(\alpha_{2}-\alpha_{1}\right) & 0 & -\frac{1}{c} \alpha_{4} \\
0 & 0 & i\left(\alpha_{2}-\alpha_{1}\right) & -2 i \alpha_{3} \\
0 & 0 & 0 & \alpha_{1}+\alpha_{2} \\
0 & 0 & 0 & 0
\end{array}\right]
\end{gathered}
$$

and

$$
\begin{gathered}
e^{\alpha_{1} a+\alpha_{2} a^{\dagger}+\alpha_{3} h+\alpha_{4} E}=e^{\left(\alpha_{1}+\alpha_{2}\right) \xi_{1}-2 i \alpha_{3} \xi_{2}-\frac{1}{c} \alpha_{4} \xi_{3}+i\left(\alpha_{2}-\alpha_{1}\right) \xi_{4}} \\
0
\end{gathered}
$$

Case (ii). $z=2 b i$ where $b \in \mathbb{R} \backslash\{0\}$ :

$$
\begin{gathered}
\alpha_{1} a+\alpha_{2} a^{\dagger}+\alpha_{3} h+\alpha_{4} E=i\left(\alpha_{2}-\alpha_{1}\right) \xi_{1}+2 i \alpha_{3} \xi_{2}-\frac{1}{b} \alpha_{4} \xi_{3}+\left(\alpha_{1}+\alpha_{2}\right) \xi_{4} \\
=\left[\begin{array}{cccc}
0 & \alpha_{1}+\alpha_{2} & 0 & -\frac{1}{b} \alpha_{4} \\
0 & 0 & \alpha_{1}+\alpha_{2} & 2 i \alpha_{3} \\
0 & 0 & 0 & i\left(\alpha_{2}-\alpha_{1}\right) \\
0 & 0 & 0 & 0
\end{array}\right]
\end{gathered}
$$

and

$$
=\left[\begin{array}{cccc}
1 & \alpha_{1}+\alpha_{2} a+\alpha_{2} a^{\dagger}+\alpha_{3} h+\alpha_{4} E & \frac{1}{2}\left(\alpha_{1}+\alpha_{2}\right)^{2} & \frac{i}{6}\left(\alpha_{1}+\alpha_{2}\right)\left(\alpha_{2}^{2}-\alpha_{1}^{2}+6 a_{3}\right)-\frac{1}{b} \alpha_{4} \\
0 & 1 & \alpha_{1}+\alpha_{2} & \frac{i}{2}\left(\alpha_{2}^{2}-\alpha_{1}^{2}+4 a_{3}\right) \\
0 & 0 & 1 & i\left(\alpha_{2}-\alpha_{1}\right) \\
0 & 0 & 0 & 1
\end{array}\right] .
$$

Case (iii). $z=2 c+2 b i$, where $b, c \in \mathbb{R} \backslash\{0\}$ : For arbitrary $\alpha, \beta \in \mathbb{R} \backslash\{0\}$ such that $\beta c-\alpha b=0$ we have 


$$
\begin{aligned}
& \alpha_{1} a+\alpha_{2} a^{\dagger}+\alpha_{3} h+\alpha_{4} E \\
& =\frac{i}{\beta}\left(\alpha_{2}-\alpha_{1}\right) \xi_{1}+2 i \alpha_{3} \xi_{2}-\frac{\beta}{b} \alpha_{4} \xi_{3}+\left(\beta\left(a_{1}+a_{2}\right)+i \alpha\left(\alpha_{1}-\alpha_{2}\right)\right) \xi_{4} \\
& =\left[\begin{array}{cccc}
0 & \beta\left(a_{1}+a_{2}\right)+i \alpha\left(\alpha_{1}-\alpha_{2}\right) & \beta\left(a_{1}+a_{2}\right)+i \alpha\left(\alpha_{1}-\alpha_{2}\right) & -\frac{\beta}{b} \alpha_{4} \\
0 & 0 & 0 & 2 i \alpha_{3} \\
0 & 0 & 0 & 0 \\
0 & 0 & 0
\end{array}\right]
\end{aligned}
$$

and

$e^{\alpha_{1} a+\alpha_{2} a^{\dagger}+\alpha_{3} h+\alpha_{4} E}=e^{\frac{i}{\beta}\left(\alpha_{2}-\alpha_{1}\right) \xi_{1}+2 i \alpha_{3} \xi_{2}-\frac{\beta}{b} \alpha_{4} \xi_{3}+\left(\beta\left(a_{1}+a_{2}\right)+i \alpha\left(\alpha_{1}-\alpha_{2}\right)\right) \xi_{4}}$

$$
=\left[\begin{array}{cccc}
1 & \left(\alpha_{1}+\alpha_{2}\right) \beta+i\left(\alpha_{1}-\alpha_{2}\right) \alpha & -\frac{1}{2}\left(\left(\alpha_{1}-\alpha_{2}\right) \alpha-i\left(\alpha_{1}+\alpha_{2}\right) \beta\right)^{2} & A_{\alpha, \beta, b}\left(\alpha_{1}, \alpha_{2}, \alpha_{3}\right) \\
0 & 1 & \left(\alpha_{1}+\alpha_{2}\right) \beta+i\left(\alpha_{1}-\alpha_{2}\right) \alpha & B_{\alpha, \beta}\left(\alpha_{1}, \alpha_{2}, \alpha_{3}\right) \\
0 & 0 & 1 & \frac{i}{\beta}\left(\alpha_{2}-\alpha_{1}\right) \\
0 & 0 & 0 & 1
\end{array}\right]
$$

where

$$
\begin{aligned}
& A_{\alpha, \beta, b}\left(\alpha_{1}, \alpha_{2}, \alpha_{3}\right) \\
& \quad=\frac{i \alpha^{2}}{6 \beta}\left(\alpha_{1}-\alpha_{2}\right)^{3}-\frac{\beta}{b} \alpha_{4}+\frac{\alpha}{3}\left(\alpha_{1}-\alpha_{2}\right)\left(\alpha_{1}^{2}-\alpha_{2}^{2}-3 \alpha_{3}\right)+\frac{i \beta}{6}\left(\alpha_{1}+\alpha_{2}\right)\left(\alpha_{2}^{2}-\alpha_{1}^{2}-6 \alpha_{3}\right)
\end{aligned}
$$

and

$$
B_{\alpha, \beta}\left(\alpha_{1}, \alpha_{2}, \alpha_{3}\right)=\left(\alpha_{1}-\alpha_{2}\right)^{2} \frac{\alpha}{2 \beta}+\frac{i}{2}\left(\alpha_{2}^{2}-\alpha_{1}^{2}+4 \alpha_{3}\right) .
$$

Defining group elements $g(u, v, w, y)=e^{u a^{\dagger}} e^{v h} e^{w a} e^{y E}$ we can use matrix multiplication to verify the group law 9.9 in all three cases.

\section{References}

[1] L. Accardi and A. Boukas, Renormalized higher powers of white noise (RHPWN) and conformal field theory, Infinite Dimensional Anal. Quantum Probab. Related Topics 9, (2006), 353-360.

[2] - - - The emergence of the Virasoro and $w_{\infty}$ Lie algebras through the renormalized higher powers of quantum white noise, International Journal of Mathematics and Computer Science 1 (2006), 315-342.

[3] - - - Renormalized higher powers of white noise and the Virasoro-Zamolodchikov- $w_{\infty}$ algebra, Reports on Mathematical Physics 61 (2008), 1-11.

[4] - , - Lie algebras associated with the renormalized higher powers of white noise, Communications on Stochastic Analysis 1 (2007), 57-69. 
[5] - - - Fock representation of the renormalized higher powers of white noise and the centerless Virasoro (or Witt)-Zamolodchikov- $w_{\infty} *$-Lie algebra, J. Phys. A: Math. Theor. 41 (2008), no. 30 .

[6] -, - Central extensions of white noise *-Lie algebras, submitted (2008).

[7] -, -, Central extensions of the Heisenberg algebra, submitted (2008).

[8] - - -, Quantum probability, renormalization and infinite dimensional *-Lie algebras, SIGMA (Symmetry, Integrability and Geometry: Methods and Applications) 5 (2009), art. 056 .

[9] L. Accardi, A. Boukas, and U. Franz, Renormalized powers of quantum white noise, Infinite Dimensional Analysis, Quantum Probability, and Related Topics 9 (2006), 129-147.

[10] L. Accardi, U. Franz, and M. Skeide, Renormalized squares of white noise and nonGaussian noises as Levy processes on real Lie algebras; Comm. Math. Phys. 228 (2002), $123-150$.

[11] L. Accardi, Y. G. Lu, and I. V. Volovich, White noise approach to classical and quantum stochastic calculi, in: Lecture Notes of the Volterra International School, Trento, 1999, Volterra Center preprint 375.

[12] L. Accardi and M. Skeide, Hilbert module realization of the square of white noise and finite difference algebras, Math. Notes 68 (2000), 683-694.

[13] - - - On the relation of the square of white noise and the finite difference algebra, Infinite Dimensional Analysis, Quantum Probability and Related Topics 3 (2000), 185-189.

[14] I. Bakas, The structure of the $W_{\text {inf }}$ algebra, Commun. Math. Phys. 134 (1990), 487-508.

[15] I. Bakas and E. B. Kiritsis, Structure and representations of the $W_{\infty}$ algebra, Prog. Theor. Phys. Supp. 102 (1991), 15.

[16] A. Boukas, An example of a quantum exponential process, Mh. Math. 112 (1991), 209-215.

[17] P. J. Feinsilver, Discrete analogues of the Heisenberg-Weyl algebra, Mh. Math. 104 (1987), 89-108.

[18] P. J. Feinsilver and R. Schott, Algebraic Structures and Operator Calculus, Volumes I and III, Kluwer, 1993.

[19] - - - Differential relations and recurrence formulas for representations of Lie groups, Stud. Appl. Math. 96 (1996), 387-406.

[20] W. Feller, Introduction to Probability Theory and its Applications, Volumes I and II, Wiley, 1971.

[21] J. Fuchs and C. Schweigert, Symmetries, Lie Algebras and Representations, Cambridge Monographs on Mathematical Physics, Cambridge University Press, 1997.

[22] R. L. Hudson and K. R. Parthasarathy, Quantum Ito's formula and stochastic evolutions, Comm. Math. Phys. 93 (1984), 301-323.

[23] S. V. Ketov, Conformal Field Theory, World Scientific, 1995.

[24] A. B. Zamolodchikov, Infinite additional symmetries in two-dimensional conformal quantum field theory, Teor. Mat. Fiz. 65 (1985), 347-359.

[25] G. Ovando, Four dimensional symplectic Lie algebras, Beiträge Algebra Geom. 47 (2006), 419-434. 
